\title{
An Analytical Theory of Piezoresistive Effects in Hall Plates with Large Contacts
}

\author{
Udo Ausserlechner 1 \\ Infineon Technologies, Villach, Austria \\ Correspondence should be addressed to Udo Ausserlechner; udo.ausserlechner@infineon.com
}

Received 13 February 2018; Accepted 21 March 2018; Published 4 June 2018

Academic Editor: Yuri Galperin

Copyright ( 2018 Udo Ausserlechner. This is an open access article distributed under the Creative Commons Attribution License, which permits unrestricted use, distribution, and reproduction in any medium, provided the original work is properly cited.

\begin{abstract}
Four-terminal transducers can be used to measure the magnetic field via the Hall effect or the mechanical stress via the piezoresistance effect. Both effects are described by an anisotropic conductivity tensor with small offdiagonal elements. This has led other authors to the conclusion that there is some kind of analogy. In both cases the output voltage depends on the geometry of the device and the size of the contacts. For Hall plates this influence is accounted for by the Hall-geometry factor. The alleged analogy proposes that the Hall-geometry factor also applies to four-terminal stress transducers. This paper shows that the analogy holds only for a limited class of devices. Moreover, it is shown that devices of different geometries may have identical magnetic field sensitivity but different mechanical stress sensitivities. Thus, shape optimization makes sense for mechanical stress sensors. In extreme cases the output voltages of vertical Hall-effect devices may have notable magnetic field sensitivity but zero mechanical stress sensitivity. As byproduct, exact analytical formulae for the equivalent resistor circuit of rectangular and circular devices with two perpendicular mirror symmetries are given. They allow for an accurate description of how mechanical stress and deformation affect the output offset voltage and the magnetic sensitivity of Hall-effect devices.
\end{abstract}

\section{Introduction}

Resistive devices with four terminals have been proposed as mechanical stress sensors for a long time[1,2]. They are called four-terminal piezotranducers [3] or van der Pauw stress sensors [4]. The very same phenomenon is observed in Hall plates at zero magnetic field, where the zero-point error (= initial offset error prior to spinning current scheme [5]) is very sensitive to mechanical stress. This was occasionally called Kanda effect [6] or pseudo-Hall effect [7]. An alternative view on the same topic is how to determine anisotropic resistivity from measurements on van der Pauw samples [8]. Over the past five decades a large number of papers have been published on various aspects of this bundle of problems. However, it seems that no analytical closed form expression was found, which relates the anisotropic conductivity to the output voltage of such a device with four contacts of arbitrary size. Conversely, the analogous problems for isotropic van der Pauw measurement at zero magnetic field and for Hall plates at small magnetic field have been solved recently [9-12]. In the following we apply similar methods to the piezoresistive effect in these devices. Thereby we use the affine transformation of van der Pauw [8] in order to replace the original device having anisotropic resistivity by an equivalent device having isotropic conductivity. For these devices with isotropic conductivity we compute the equivalent resistor circuit (ERC), which describes the behavior of the devices under the action of mechanical stress and magnetic field. For the ERC of rectangular devices we use our own results of former works [9-12], whereas we derive new formulae for the ERC of circular devices.

The outline of this work is as follows. Section 2 sums up basic phenomenological facts of the Hall effect and of piezoresistivity as they are known in the literature. The goal is to contrast similarities against differences of both effects. Section 3 briefly repeats the affine transformation of van der Pauw and specifies it for (100)-planes of cubic 
crystals, like silicon. With these formulae it becomes apparent that vertical Hall-effect devices are very slightly affected by mechanical stress. Section 4 elaborates on how rectangular and circular devices respond to biaxial inplane stress. There we make ample use of the ERC as it was developed in prior works $[9,10]$. Section 4 closes with a comparison of several device shapes versus Wheatstone bridge circuits of resistor stripes in orthogonal directions: which one has the largest sensitivity to mechanical stress? Section 5 discusses the effect of biaxial inplane stress on the Hall-geometry factor of Hall plates. This leads to a drift of magnetic sensitivity versus mechanical stress, which is caused by piezoresistance and not by piezo-Hall effect. Finally, Section 6 compares the proposed analytical theory with results of other authors.

\section{Similarities of Piezoresistance and Hall Effect}

In a thin plate-like material with isotropic ohmic resistivity the Hall effect is described by the relation $\vec{E}=\underline{\underline{\rho}} \overrightarrow{\underline{J}}$ between electric field $\vec{E}$ and current density $\vec{J}$ with the odd symmetric resistivity tensor in $(x, y)$-coordinates

$$
\underline{\underline{\rho}}=\rho_{0}\left(\begin{array}{cc}
1 & \mu_{H} B_{z} \\
-\mu_{H} B_{z} & 1
\end{array}\right)
$$

$\rho_{0}$ is the resistivity at zero magnetic field, $\mu_{H}>0$ is the Hall mobility, and $B_{z}$ is the magnetic flux density component perpendicular to the Hall plate, which lies in the $(x, y)$ plane [13]. The inverse relation $\vec{J}=\underline{\kappa} \vec{E}$ uses the conductivity tensor

$$
\underline{\underline{\kappa}}=\underline{\underline{\rho}}^{-1}=\frac{\kappa_{0}}{1+\mu_{H}^{2} B_{z}^{2}}\left(\begin{array}{cc}
1 & -\mu_{H} B_{z} \\
\mu_{H} B_{z} & 1
\end{array}\right) .
$$

$\kappa_{0}=1 / \rho_{0}$ is the conductivity at zero magnetic field. Equations (1a) and (1b) assume negative charge carriers. Alternatively to $\mu_{H}$ the literature also uses the Hall coefficient $C_{H}=$ $\operatorname{sgn}(q) \rho_{0} \mu_{H}$, where $\operatorname{sgn}(q)$ is the sign of the charge carriers. The static electric field is longitudinal, i.e., its curl vanishes. Therefore it can be expressed as the negative gradient of a scalar electric potential $\vec{E}=-\nabla \phi$. In the quasistatic case the divergence of the current density vanishes $\vec{\nabla}=0$. Combining these equations with (1b) gives

$$
\frac{\partial^{2} \phi}{\partial x^{2}}+\mu_{H} B_{z}\left(\frac{\partial^{2} \phi}{\partial x \partial y}-\frac{\partial^{2} \phi}{\partial y \partial x}\right)+\frac{\partial^{2} \phi}{\partial y^{2}}=0
$$

whereby we assumed that $\kappa_{0}, \mu_{H}$, and $B_{z}$ are constant versus spatial coordinates. The mixed-order terms $\partial^{2} \phi / \partial x \partial y=$ $\partial^{2} \phi / \partial y \partial x$ cancel out and therefore (2) is the Laplace equation.
Thus, the Hall effect does not enter (2); with and without magnetic field it is the same partial differential equation for the potential. The magnetic field affects only the boundary conditions: the potential at the contacts or the current flow via the contacts is forced by the external circuitry. At the isolating boundary the current density must flow parallel to the boundary and this means $J_{n}=0$ or in other words $E_{n} / E_{p}=(\partial \phi / \partial n) /(\partial \phi / \partial t)= \pm \mu_{H} B_{z}$ (where $n$ and $t$ denote normal and tangential direction and the plus/minus sign depends on the direction of the magnetic field, the sign of the charge carriers, and if the boundary is left or right to the current flow). In other words, at the isolating boundary there is the Hall angle $\theta_{H}=\arctan \left(\mu_{H} B_{z}\right)$ between the electric field and the boundary. To sum up, the potential $\phi$ in a Hall plate and the output voltage $V_{\text {out }}$ of a Hall plate can be computed by solving the Laplace equation and satisfying these boundary conditions. For Hall plates with two perpendicular mirror symmetries this was done in the seminal work [14] and the result is

$$
V_{\text {out }}=S_{i} I_{\text {in }} B_{z} \quad \text { with } S_{i}=R_{\text {sh }} \mu_{H} G_{H} \text {, }
$$

where $S_{i}$ is the current related magnetic sensitivity, $R_{\mathrm{sh}}$ is the sheet resistance (equal to one over the product of conductivity times thickness of the Hall plate), $I_{\text {in }}$ is the current flowing through the Hall plate, and $G_{H}$ is the Hall geometry factor. At small magnetic field we write $G_{H} \rightarrow G_{H 0}$ and then the Hall geometry factor depends only on the lateral geometry (= the layout) of the Hall plate, not on its thickness and not on any material parameters. We can compute $G_{H 0}$ for any geometry of Hall plates from scratch (in rare cases analytically and in general numerically), but it is simpler to measure the input and output numbers of squares as shown in $[9,10]$ and to use the following formula (which is derived in [12]):

$$
\begin{aligned}
G_{H 0}= & \frac{1}{K\left(\sqrt{L\left(\lambda_{p}\right)}\right) K\left(\sqrt{L\left(\lambda_{f}\right)}\right)} \\
& \cdot \int_{\alpha=0}^{\pi / 2} \frac{F\left(\sin \alpha, \sqrt{1-L\left(\lambda_{p}\right)}\right)}{\sqrt{\sin ^{2} \alpha+L\left(\lambda_{f}\right) \cos ^{2} \alpha}} d \alpha
\end{aligned}
$$

Thereby $F(w, k)=\int_{0}^{w}\left(1-\alpha^{2}\right)^{-1 / 2}\left(1-k^{2} \alpha^{2}\right)^{-1 / 2} d \alpha$ is the incomplete elliptic integral of the first kind, $K(k)=F(1, k)$ is the complete elliptic integral of the first kind, and $L(y)$ is the modular lambda function defined by $L\left(K^{\prime}(k) / K(k)\right)=$ $k^{2}$ for $0 \leq k \leq 1$ and $K^{\prime}(k)=K\left(\sqrt{1-k^{2}}\right)$. The input number of squares is $\lambda_{f}=R_{\text {in }} / R_{\text {sh }}$ and the output number of squares is $\lambda_{p}=R_{\text {out }} / R_{\mathrm{sh}}$, whereby the input resistance $R_{\text {in }}$ is measured between the two current supplying contacts of a Hall plate (while the sense contacts are floating) and the output resistance $R_{\text {out }}$ is measured between the two sense contacts (while the other two contacts are floating). The role of input and output contacts is arbitrary $-G_{H 0}$ does not change if we swap it [12]. Equation (4) expresses the Hall 
geometry factor as a function of input and output numbers of squares without need to know any geometrical details of the device. This means that Hall plates with different geometry have identical $G_{H 0}$ if they have identical $\lambda_{f}$ and $\lambda_{p}$. The reason why different geometries may have the same number of squares and the same Hall output voltage at identical supply current is that we can transform one into the other via conformal mapping [14]. Since the conformal mapping is done by analytic functions which satisfy CauchyRiemann differential equations, the mapping functions are also solutions of the Laplace equations. Therefore, if we have a solution of the potential in one Hall plate, we can apply a conformal transformation to this geometry and to the solution. Thereby the same current will flow through the transformed device and the same potentials will appear at its contacts. Consequently, we can study a specific device geometry (e.g., a circular disk) and we can be sure that there is no other ingeniously shaped device boundary (e.g., Greek cross or octagon...) that leads to better device performance. This was already noted by Wick in [14]. Of course this holds only within the limited scope of linear electrostatic theory; beyond that scope devices will be different: different shapes lead to more or less homogeneous electric fields and therefore to more or less pronounced velocity saturation effects. Although their total power may be identical, the spatial distribution of power density differs and this leads to different temperature distributions. The temperature coefficient of the resistivity acts back on the current distribution. Finally the contacts may have different temperatures, which leads to different thermoelectric voltages and different residual offsets after spinning current schemes are applied [15].

In the case there is piezoresistance at zero magnetic field, the conductivity tensor $\underline{\underline{\kappa}}$, and its inverse the resistivity tensor $\underline{\underline{\rho}}$ becomes anisotropic due to mechanical stress

$$
\underline{\underline{\kappa}}=\left(\begin{array}{ll}
\kappa_{x x} & \kappa_{x y} \\
\kappa_{x y} & \kappa_{y y}
\end{array}\right)=\underline{\underline{\rho}}^{-1}=\left(\begin{array}{ll}
\rho_{x x} & \rho_{x y} \\
\rho_{x y} & \rho_{y y}
\end{array}\right)^{-1}
$$

The significant difference between (1a), (1b), and (5) is that the conductivity tensor of the Hall effect is odd symmetric, whereas it is even symmetric for the piezoresistance. In the language of nonequilibrium thermodynamics both Hall effect and piezoresistance are irreversible dissipative processes, but the relation between current density and electric field is reciprocal for piezoresistance whereas it is antireciprocal for the Hall effect [16]. Therefore, a rotation of the coordinate system around the $z$-axis does not change the Hall-resistivity tensor (1a), yet it changes the piezoresistance resistivity tensor (5) in such a way that for certain orientations (the principal axes) the elements in the minor diagonal even vanish. In network theory it is well known that any reciprocal (i.e., symmetric) impedance matrix of a linear, lumped, and passive N-port can be realized by a network of

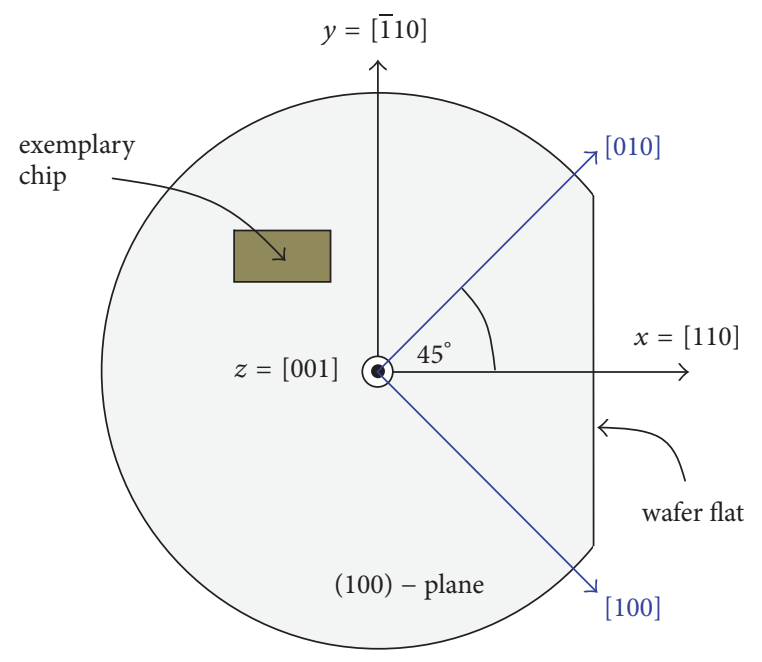

FIGURE 1: Reference frame $\langle 100\rangle$ of the single crystal and chip reference frame $\Sigma=(x, y, z)$ for a (100)-silicon wafer.

resistors, inductances, capacitors, and ideal transformers [17], however a nonreciprocal impedance matrix needs at least one additional linear, lumped, passive element: the gyrator [18]. Instead of a gyrator one may also use the series connection of two current controlled voltage sources (CCVS) or the parallel connection of two voltage controlled current sources (VCCS). Therefore the equivalent lumped circuit of a Hall effect device comprises resistors and at least one gyrator $[19,20]$, whereas the equivalent lumped circuit of a piezoresistive device consists only of resistors. Hence, the gyrator makes an essential difference between piezoresistance devices and Hall effect devices, and this already denies any perfect analogy between piezoresistance and Hall effect.

In a conventional plastic encapsulated package the surface of a semiconductor chip is exposed to inplane mechanical stress [21, 22]. For inplane normal stress $\sigma_{x x}, \sigma_{y y}$ and inplane shear stress components $\sigma_{x y}$ on a (100) silicon chip it holds [23]

$$
\begin{aligned}
\rho_{x x} & =\rho_{0}\left(1+\frac{\pi_{11}+\pi_{12}+\pi_{44}}{2} \sigma_{x x}\right. \\
& \left.+\frac{\pi_{11}+\pi_{12}-\pi_{44}}{2} \sigma_{y y}\right), \\
\rho_{y y} & =\rho_{0}\left(1+\frac{\pi_{11}+\pi_{12}-\pi_{44}}{2} \sigma_{x x}\right. \\
& \left.+\frac{\pi_{11}+\pi_{12}+\pi_{44}}{2} \sigma_{y y}\right), \\
\rho_{x y} & =\rho_{0}\left(\pi_{11}-\pi_{12}\right) \sigma_{x y}
\end{aligned}
$$

with $\pi_{11}, \pi_{12}$, and $\pi_{44}$ being the three piezoresistive coefficients of the cubic $\mathrm{m} 3 \mathrm{~m}$ single crystal. Thereby the $x$ - and $y$-axes of the chip are aligned along $\langle 110\rangle$-crystal directions as shown in Figure 1 (see also [24]). 
Computing the conductivities from (6a) to (6c) gives

$$
\begin{aligned}
& \kappa_{x x}=\frac{\rho_{0}}{\rho_{\text {iso }}^{2}}\left(1+\frac{\pi_{11}+\pi_{12}-\pi_{44}}{2} \sigma_{x x}+\frac{\pi_{11}+\pi_{12}+\pi_{44}}{2} \sigma_{y y}\right), \\
& \kappa_{y y}=\frac{\rho_{0}}{\rho_{\text {iso }}^{2}}\left(1+\frac{\pi_{11}+\pi_{12}+\pi_{44}}{2} \sigma_{x x}+\frac{\pi_{11}+\pi_{12}-\pi_{44}}{2} \sigma_{y y}\right), \\
& \kappa_{x y}=\frac{\rho_{0}}{\rho_{\text {iso }}^{2}}\left(\pi_{12}-\pi_{11}\right) \sigma_{x y}, \\
& \rho_{\text {iso }}=\sqrt{\rho_{x x} \rho_{y y}-\rho_{x y}^{2}}=\rho_{0} \sqrt{\left(1+\frac{\pi_{11}+\pi_{12}}{2}\left(\sigma_{x x}+\sigma_{y y}\right)\right)^{2}-\left(\frac{\pi_{44}}{2}\right)^{2}\left(\sigma_{x x}-\sigma_{y y}\right)^{2}-\left(\pi_{11}-\pi_{12}\right)^{2} \sigma_{x y}^{2} .}
\end{aligned}
$$

$\rho_{\text {iso }}$ is the determinant of the resistivity matrix, and its name will become apparent later in $(12 \mathrm{a})-(12 \mathrm{c})$. For $\sigma_{x x}=\sigma_{y y}=0$ we get

$$
\underline{\underline{\kappa}}=\rho_{0}^{-1}\left(1-\delta_{12}^{2}\right)^{-1}\left(\begin{array}{cc}
1 & \delta_{12} \\
\delta_{12} & 1
\end{array}\right)
$$

with $\delta_{12}=\left(\pi_{12}-\pi_{11}\right) \sigma_{x y}$ and $\left|\delta_{12}\right| \ll 1$. In the general case $\sigma_{x x}, \sigma_{y y}, \sigma_{x y} \neq 0$ we can apply the Mohr circle construction [25] to the second-rank conductivity tensor in

$$
\underline{\underline{\kappa}}=\frac{1}{2}\left(\begin{array}{c}
\kappa_{x x}+\kappa_{y y} \\
\pm\left(\operatorname{sgn} \kappa_{x y}\right) \sqrt{\left(\kappa_{x x}-\kappa_{y y}\right)^{2}+\left(2 \kappa_{x y}\right)^{2}}
\end{array}\right.
$$$$
\left.\begin{array}{c} 
\pm\left(\operatorname{sgn} \kappa_{x y}\right) \sqrt{\left(\kappa_{x x}-\kappa_{y y}\right)^{2}+\left(2 \kappa_{x y}\right)^{2}} \\
\kappa_{x x}+\kappa_{y y}
\end{array}\right)
$$

(5): we rotate the $(x, y)$-coordinate system $\Sigma$ by an angle $\tilde{\varphi}$ in mathematically positive direction (counterclockwise)

$$
\tan (2 \widetilde{\varphi})=\frac{\kappa_{y y}-\kappa_{x x}}{2 \kappa_{x y}}=\frac{\pi_{44}}{\pi_{12}-\pi_{11}} \frac{\sigma_{x x}-\sigma_{y y}}{2 \sigma_{x y}}
$$

to obtain the rotated coordinate system $\widetilde{\Sigma}(\tilde{x}, \tilde{y})[26]$. In $\widetilde{\Sigma}$ the conductivity tensor has equal values on the main diagonal and maximum value on the minor diagonal

conductivity tensor being even symmetric. As for the boundary conditions they are similar to the Hall effect: the potential has constant values at the contacts, whereas it holds $E_{n} / E_{p}=$ $(\partial \phi / \partial n) /(\partial \phi / \partial t)=\widetilde{\delta}_{12}$ at isolation boundaries parallel to $\tilde{x}$ axis or $\tilde{y}$-axis. So $\widetilde{\delta}_{12}$ seems to take over the role of $\tan \theta_{H}=$ $\mu_{H} B_{z}$ - at least at these isolation boundaries. However, inside the sample there is a striking difference between Hall effect and piezoresistance $[27,28]$ : inside the Hall-effect region the angle between electric field vector $\vec{E}$ and current density vector $\vec{J}$ is homogeneous versus spatial position and equal to the Hall angle $\angle\{\vec{E}, \vec{J}\}=\theta_{H}=\arctan \left(\mu_{H} B_{z}\right)$, whereas in the piezoresistive region in general the angle between $\vec{E}$ and $\vec{J}$ is inhomogeneous versus spatial position $\angle\{\vec{E}, \vec{J}\}=$ $\arctan \left(\widetilde{\delta}_{12}\left(E_{\tilde{x}}^{2}-E_{\tilde{y}}^{2}\right) /\left(E_{\tilde{x}}^{2}+E_{\tilde{y}}^{2}+2 \widetilde{\delta}_{12} E_{\tilde{x}} E_{\tilde{y}}\right)\right)$.

The similarities between Hall effect and piezoresistance led some authors to presume an analogy [3, 29-31], since $\left|\widetilde{\delta}_{12}\right| \ll 1$ it is tempting to drop the mixed derivatives in (11). Then the piezoresistive potential would become a solution of Laplace's equation just like the Hall potential and since both fulfill the same boundary conditions, we would only have to replace $\mu_{H} B_{z}$ by $\widetilde{\delta}_{12}$ to fulfill the skew-derivative boundary conditions. Then the output voltage of a van der Pauw stress
(11) is not Laplace's equation, because the mixed derivative does not vanish and this is a consequence of the piezoresistive

$$
\frac{\partial^{2} \phi}{\partial \tilde{x}^{2}}+2 \widetilde{\delta}_{12} \frac{\partial^{2} \phi}{\partial \tilde{x} \partial \tilde{y}}+\frac{\partial^{2} \phi}{\partial \widetilde{y}^{2}}=0
$$

So in any case we get a symmetric conductivity tensor with effect we get a second-order partial differential equation for the electric potential 


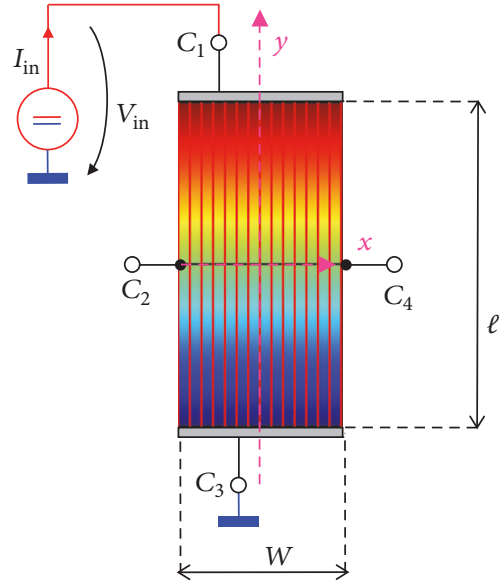

(a)

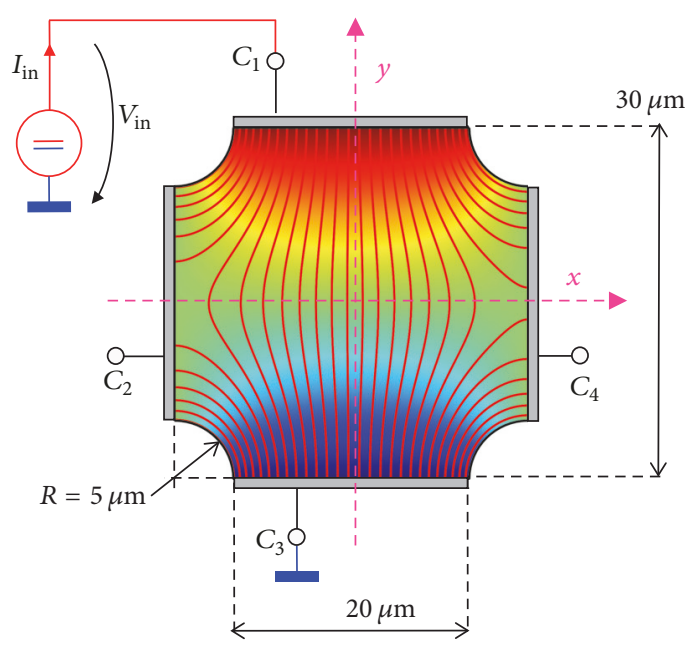

(b)

Figure 2: (a) Rectangular device with large supply contacts $C_{1}-C_{3}$ and point-sized sense contacts $C_{2}-C_{4}$ with potential distribution and current streamlines at $\sigma_{x y}=1 \mathrm{MPa}$. At zero stress it has two squares input resistance $\ell / W=2$ and according to (4) this gives $G_{H 0}=0.93006$. (b) Cross-shaped device with large supply contacts $C_{1}-C_{3}$ and large sense contacts $C_{2}-C_{4}$ with potential distribution and current streamlines at $\sigma_{x y}=1 \mathrm{MPa}$. At zero stress it has 1.0359 squares input resistance and according to (4) this gives $G_{H 0}=0.45364$. The plots were obtained by a finite element simulation (FEM) with commercial software code COMSOL MULTIPHYSICS. This FEM simulation gives a stress output signal of device (a) which differs only $0.4 \mathrm{ppm}$ from $V_{\text {out }}=R_{\mathrm{sh}} I_{\mathrm{in}}\left(\pi_{12}-\pi_{11}\right) \sigma_{x y} G_{H 0}$ from [2]. An FEM calculation of device (b) gives an output signal that is $57 \%$ larger than $V_{\text {out }}=R_{\mathrm{sh}} I_{\text {in }}\left(\pi_{12}-\pi_{11}\right) \sigma_{x y} G_{H 0}$.

sensor would be $V_{\text {out }} \cong R_{\text {sh }} I_{\text {in }} \widetilde{\delta}_{12} G_{H 0}$ analogous to (3), whereby the $\cong$ sign denotes small stress and there we may neglect the stress dependence of $R_{\mathrm{sh}}$. If both normal and shear stress are present in (10b) they would mix quadratically. If only inplane shear stress is present on (100)-silicon we would get $V_{\text {out }} \cong R_{\text {sh }} I_{\text {in }}\left(\pi_{12}-\pi_{11}\right) \sigma_{x y} G_{H 0}$. These relations may hold for certain device geometries, like the one in [2] with small sense contacts and homogeneous current density (which is shown in Figure 2(a)), or for devices with $G_{H 0} \cong 1$ (as in [32]), but they do not hold in the general case. Figure 2(b) shows a device geometry with $90^{\circ}$ symmetry and large contacts, where these simple formulae lead to an error of $36 \%$ in the output voltage when compared to an accurate finite element simulation.

Where does this error come from? In (11) we had to neglect the mixed derivative in order to obtain the analogy with the Hall effect, and this was not allowed. Of course we may neglect the $\widetilde{\delta}_{12}$-term in (11) if we want to compute the potential $\phi$ up to zero-th order in $\widetilde{\delta}_{12}$. However, if we compute the output voltage of the pseudo-Hall device, we subtract the potential at both output contacts in order to get the small imbalance of potential due to $\widetilde{\delta}_{12}$. Hence, this output voltage is proportional to $\widetilde{\delta}_{12}$; it is of first-order in $\widetilde{\delta}_{12}$ and so we must not neglect terms in (11) which are linear in $\widetilde{\delta}_{12}$. Therefore in general we cannot use the Hall geometry factor $G_{H 0}$ for van der Pauw stress sensors. However, in the special case of Figure 2(a) at small stress the potential is constant versus $x$. Therefore the mixed derivative in (11) vanishes and (11) becomes the Laplace equation. For this particular symmetry (11) and (2) are identical and the piezoresistive output is analogous to the Hall output when we replace $\widetilde{\delta}_{12}$ by $\mu_{H} B_{z}$.
Figures $3($ a) and $3(\mathrm{~b})$ show the angle $\angle\{\vec{E}, \vec{J}\}$ between electric field and current density for the device of Figure 2(b) operated in the same way as in Figure 2(b). However in Figure 3(a) there is small shear stress $\left(\pi_{12}-\pi_{11}\right) \sigma_{x y}=$ $\tan 0.446^{\circ}$ and no magnetic field whereas in Figure $3(\mathrm{~b})$ there is large magnetic field $\mu_{H} B_{z}=\tan 51.078^{\circ}$ and no mechanical stress. Obviously, in Figure $3($ a) the angle $\angle\{\vec{E}, \vec{J}\}$ is small but inhomogeneous: it varies $\pm 100 \%$ from $-0.446^{\circ}$ at the supply contacts to $+0.446^{\circ}$ at the sense contacts. Conversely, in Figure $3(\mathrm{~b})$ the angle $\angle\{\vec{E}, \vec{J}\}$ is large and perfectly homogeneous $\left(51.078^{\circ}\right)$. Figures $4(\mathrm{a})$ and $4(\mathrm{~b})$ show the same angle $\angle\{\vec{E}, \vec{J}\}$ for the device of Figure 2(a). In Figure 4(a) there is again small shear stress $\left(\pi_{12}-\pi_{11}\right) \sigma_{x y}=\tan 0.446^{\circ}$ at zero magnetic field and in Figure 4(b) there is a large Hall angle $\theta_{H}=51.078^{\circ}$ at zero stress. Figure $4(\mathrm{~b})$ shows again a perfectly homogeneous large Hall angle. In Figure 4(a) the angle $\angle\{\vec{E}, \vec{J}\}$ is within a very narrow range of $-0.445766^{\circ}$ and $-0.445752^{\circ}$; nevertheless the color coding reveals a systematic pattern of this angle. This is a striking contrast to Figure 3(a). The specific geometry of the device of Figure 4(a) gives a homogeneous current density and thus a homogeneous angle $\angle\{\vec{E}, \vec{J}\}$ at small mechanical stress just like for the Hall effect. This seems to be the deeper reason why one may use the Hall geometry factor in Figure 4(a) to compute the output voltage at shear stress, although this is not allowed for other device geometries like in Figure 3(a). At larger stress on the device in Figure 4(a) the spatial dependence of $\angle\{\vec{E}, \vec{J}\}$ gets more pronounced, yet this inhomogeneity is still smaller than for Figure 3(a) and the piezoresistive angle $\angle\{\vec{E}, \vec{J}\}$ will never change sign as in Figure 3(a). 


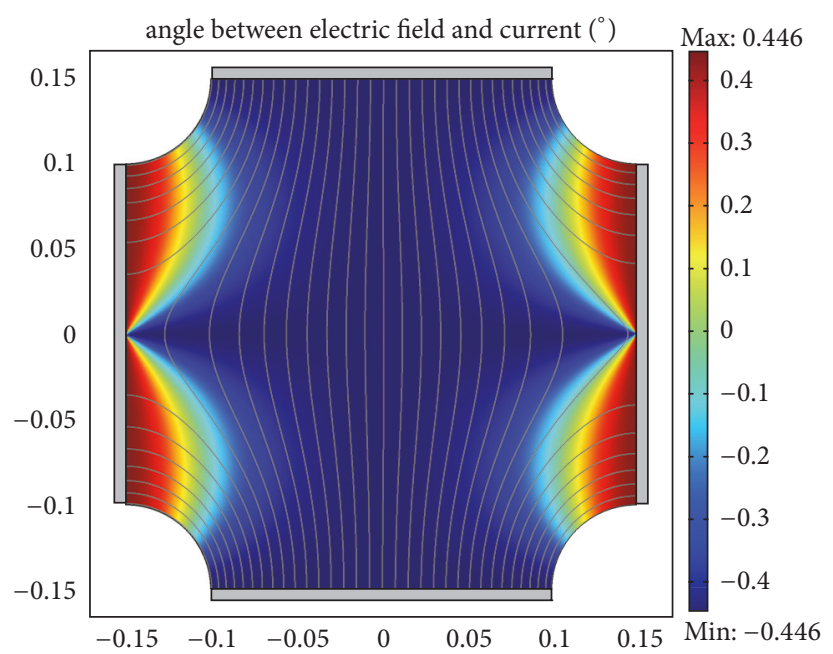

(a)

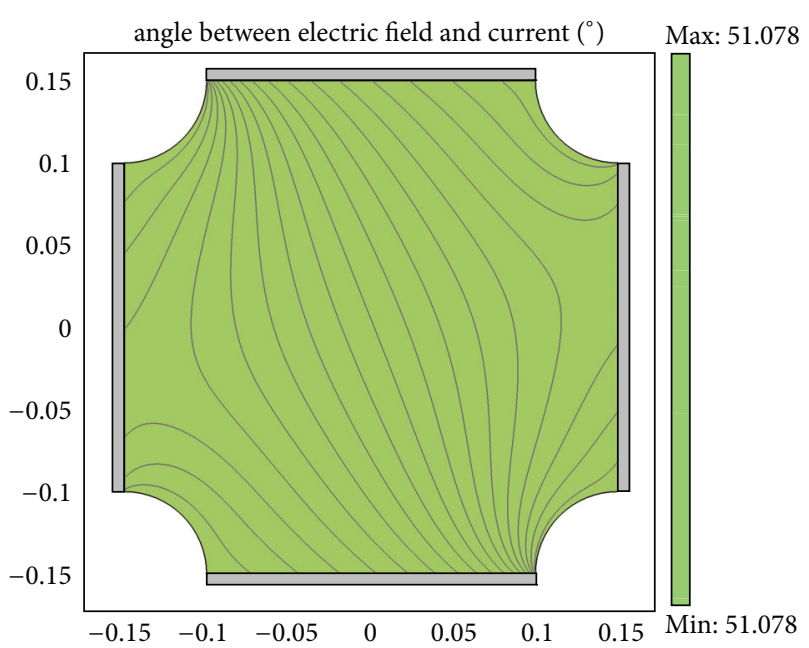

(b)

Figure 3: (a, b) Angle between electric field and current density in a device of Figure 2(b) operated like in Figure 2(b). The plots were obtained by FEM simulations with COMSOL MULTIPHYSICS. In (a) we have zero magnetic field and small mechanical stress $\left(\pi_{12}-\pi_{11}\right) \sigma_{x y}=$ $\tan 0.446^{\circ}$. In (b) we have zero stress and even large magnetic field with $51.078^{\circ}$ Hall angle. As predicted by [28] the Hall angle is perfectly homogeneous throughout the device (b), whereas in the piezoresistive case (a) the angle $\angle\{\vec{E}, \vec{J}\}$ varies between $-0.446^{\circ}$ at the supply contacts and in the major portion of the device and $+0.446^{\circ}$ at the sense contacts. Therefore the analogy [2] between Hall effect and piezoresistive effect does not apply in this case.

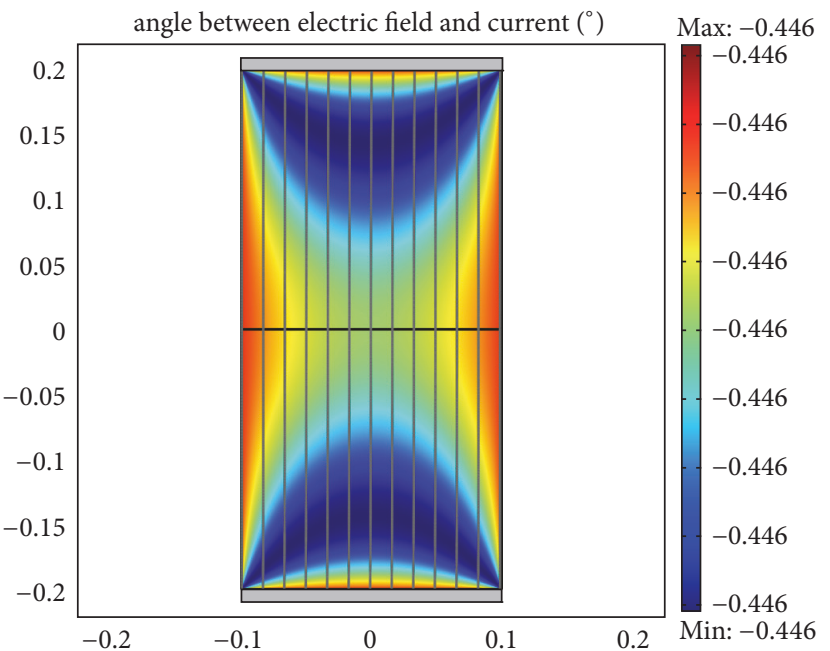

(a)

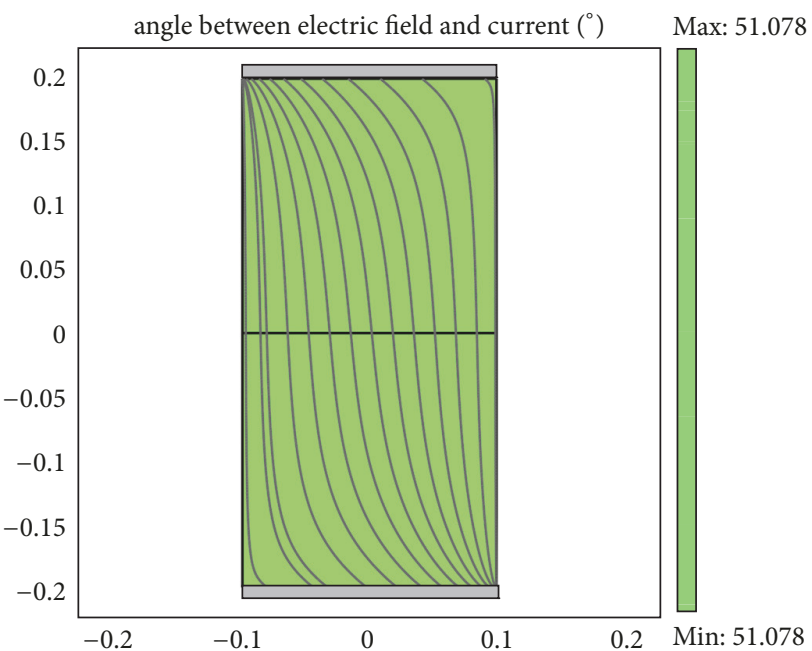

(b)

Figure 4: (a, b) Angle between electric field and current density in a device of Figure 2(a) operated like in Figure 2(a). The plots were obtained by FEM simulations with COMSOL MULTIPHYSICS. In (a) we have zero magnetic field and small mechanical stress $\left(\pi_{12}-\pi_{11}\right) \sigma_{x y}=$ $\tan 0.446^{\circ}$. In (b) we have zero stress and even large magnetic field with $51.078^{\circ}$ Hall angle. As predicted by [28] the Hall angle is perfectly homogeneous throughout the device (b), but surprisingly in the piezoresistive case (a) the angle $\angle\{\vec{E}, \vec{J}\}$ is also highly homogeneous (it varies only between $-0.445766^{\circ}$ and $-0.445752^{\circ}$ ). This seems to be the reason why the analogy [2] between Hall effect and piezoresistive effect works for this device.

\section{The Affine Transformation}

Since (11) is not Laplace's equation, we cannot directly apply a conformal transformation to solve it. However, (11) can be transformed into Laplace's equation by an affine transformation $\Sigma(x, y) \rightarrow \Sigma^{\prime}\left(x^{\prime}, y^{\prime}\right)$. This procedure has been worked out in $[8,24,33]$ (the affine transformation is also used in [34]; however, it seems that conductivities in $x$ - and $y$-directions are mixed up. [7] uses again a different transformation which does not seem to comply with 
(12a)-(12c).). We summarize it here briefly in a notation that will we used in the rest of this work.

$$
\begin{aligned}
&\left(\begin{array}{l}
x^{\prime} \\
y^{\prime}
\end{array}\right)=\underline{\underline{M}}^{\text {piezo }}\left(\begin{array}{l}
x \\
y
\end{array}\right) \\
& \text { with } \underline{\underline{M}}^{\text {piezo }}=\left(\begin{array}{cc}
\frac{1}{\sqrt{\rho_{\text {iso }} \kappa_{x x}}} & 0 \\
-\kappa_{x y} \sqrt{\frac{\rho_{\text {iso }}}{\kappa_{x x}}} & \sqrt{\rho_{\text {iso }} \kappa_{x x}}
\end{array}\right)
\end{aligned}
$$

In the transformed geometry the equivalent device has an isotropic conductivity

$$
\begin{aligned}
\underline{\kappa^{\prime}} & =\frac{1}{\rho_{\text {iso }}}\left(\begin{array}{ll}
1 & 0 \\
0 & 1
\end{array}\right), \\
\rho_{\text {iso }} & =\sqrt{\rho_{x x} \rho_{y y}-\rho_{x y}^{2}}=\frac{1}{\kappa_{\text {iso }}}=\frac{1}{\sqrt{\kappa_{x x} \kappa_{y y}-\kappa_{x y}^{2}}}
\end{aligned}
$$

The potentials in corresponding points before and after the transformation are identical: $\phi(x, y)=\phi^{\prime}\left(x^{\prime}, y^{\prime}\right)$. The matrix $\underline{M}^{\text {piezo }}$ is chosen such that the currents through corresponding contacts remain constant under the transformation, because only then the transformation preserves the resistances. Also the total power dissipation in the device and the power density are not affected by the transformation. This implies that the thickness and the area of the device remain constant under the transformation [24].

Inserting (7a)-(7c) into (12a) and developing into a MacLaurin series for small stress terms gives the approximation

$$
\begin{aligned}
& \underline{\underline{M}}^{\text {piezo }} \\
& \cong\left(\begin{array}{cc}
1+\frac{\pi_{44}\left(\sigma_{x x}-\sigma_{y y}\right)}{4} & 0 \\
\left(\pi_{11}-\pi_{12}\right) \sigma_{x y} & 1-\frac{\pi_{44}\left(\sigma_{x x}-\sigma_{y y}\right)}{4}
\end{array}\right), \\
& \rho_{\text {iso }} \cong \rho_{0}\left(1+\frac{\pi_{11}+\pi_{12}}{2}\left(\sigma_{x x}+\sigma_{y y}\right)\right), \\
& \rho_{\text {iso }} \kappa_{x x} \cong 1-\frac{\pi_{44}}{2}\left(\sigma_{x x}-\sigma_{y y}\right) .
\end{aligned}
$$

The affine transformation (12a), (12b), and (12c) applies to all crystal classes; however, (13a), (13b), and (13c) use (7a), (7b), $(7 \mathrm{c})$, and $(7 \mathrm{~d})$ and therefore they apply only to (100)-planes of cubic crystal classes (e.g., silicon).
Note that an affine transformation is definitely not a conformal transformation: conformal transformations preserve the angles between two curves, whereas affine transformations do not, because they skew the geometry. Equation (13a) shows that the geometry is skewed for shear stress. The difference of normal inplane stresses scales the $x$ - and $y$-axes differently. This also skews most devices except those ones with edges parallel to $x-y$-axes. The sum of normal inplane stresses scales the equivalent isotropic conductivity. Isotropic stress $\sigma_{x x}=\sigma_{y y}$ does not change the shape of the equivalent device; it only changes $\rho_{0} \rightarrow \rho_{\text {iso }}$.

So far we neglected the pure deformation of the geometry (= strain) caused by the stress on the device. This is commonly justified because for many materials the piezoresistance effect dominates. However, the affine transformation (12a) already describes a deformation of the device, and so we can add the strain terms without unduly increasing the complexity of the calculation. A homogeneous two-dimensional strain with zero displacement of the origin is described by the transformation [35]

$$
\begin{aligned}
\left(\begin{array}{l}
x^{\prime} \\
y^{\prime}
\end{array}\right)=\underline{\underline{M}}^{\text {strain }}\left(\begin{array}{l}
x \\
y
\end{array}\right) & \text { with } \underline{\underline{M}}^{\text {strain }}=\left(\begin{array}{cc}
1+\varepsilon_{x x} & \varepsilon_{x y} \\
\varepsilon_{x y} & 1+\varepsilon_{y y}
\end{array}\right)
\end{aligned}
$$

whereby $\varepsilon_{x x}, \varepsilon_{y y}$, and $\varepsilon_{x y}$ are the tensor strains (as opposed to the engineering strains [36]). The strain is related to the stress via the fourth rank compliance tensor, which in silicon has only three nonvanishing terms $s_{11}=7.68 \times 10^{-12} \mathrm{~Pa}, s_{12}=$ $-2.14 \times 10^{-12} \mathrm{~Pa}$, and $s_{44}=12.6 \times 10^{-12} \mathrm{~Pa}$ [37]. Transforming the tensors into the chip coordinate system $\Sigma=(x, y, z)$ of Figure 1 gives

$$
\begin{aligned}
& \varepsilon_{x x}=\left(s_{11}+s_{12}\right) \frac{\sigma_{x x}+\sigma_{y y}}{2}+s_{44} \frac{\sigma_{x x}-\sigma_{y y}}{4}, \\
& \varepsilon_{y y}=\left(s_{11}+s_{12}\right) \frac{\sigma_{x x}+\sigma_{y y}}{2}-s_{44} \frac{\sigma_{x x}-\sigma_{y y}}{4}, \\
& \varepsilon_{x y}=\left(s_{11}-s_{12}\right) \sigma_{x y}, \\
& \varepsilon_{z z}=s_{12}\left(\sigma_{x x}+\sigma_{y y}\right) .
\end{aligned}
$$

The last equation (15d) means that the thickness of the conducting region changes, too. We take account of this by keeping the unstrained thickness but changing the resistivity accordingly $\rho_{\text {iso }} \rightarrow \rho_{\text {iso }} /\left(1+\varepsilon_{z z}\right)$. The combined effect of piezoresistance and strain is obtained by multiplying both matrices $\underline{\underline{M}}^{\text {piezo }}$ and $\underline{\underline{M}}^{\text {strain }}$, whereby the order of multiplication is irrelevant for small stress. 


$$
\begin{aligned}
& \underline{\underline{M}}^{\text {strain }} \underline{\underline{M}}^{\text {piezo }} \\
& \cong\left(\begin{array}{cc}
1+\left(\pi_{44}+s_{44}\right) \frac{\sigma_{x x}-\sigma_{y y}}{4}+\left(s_{11}+s_{12}\right) \frac{\sigma_{x x}+\sigma_{y y}}{2} & \left(s_{11}-s_{12}\right) \sigma_{x y} \\
\left(\pi_{11}-\pi_{12}\right) \sigma_{x y}+\left(s_{11}-s_{12}\right) \sigma_{x y} & 1-\left(\pi_{44}+s_{44}\right) \frac{\sigma_{x x}-\sigma_{y y}}{4}+\left(s_{11}+s_{12}\right) \frac{\sigma_{x x}+\sigma_{y y}}{2}
\end{array}\right), \\
& \rho_{\mathrm{iso}} \cong \rho_{0} \frac{1+\left(\pi_{11}+\pi_{12}\right)\left(\sigma_{x x}+\sigma_{y y}\right) / 2}{1+s_{12}\left(\sigma_{x x}+\sigma_{y y}\right)}
\end{aligned}
$$

In the literature of the Hall geometry factor it is a common technique to map finite device geometries like disks, squares, rectangles, crosses, or octagons via conformal mapping onto the infinite upper half space of the $(x, y)$ plane $[11,14,38]$. If the original devices have contacts only at their perimeter, the conformal mapping transfers these contacts onto the $x$-axis boundary. Let us for a moment assume an original device with an arbitrary number of contacts on the $x$-axis, its active region being the entire upper half plane with anisotropic resistivity (6a), (6b), and (6c). We can transform it via affine transformation (12a), (12b), and (12c) into an equivalent problem with isotropic resistivity $\rho_{\text {iso }}$. However, since all contacts are on the $x$ axis and the geometry is infinite, matrix $\underline{M}$ just scales the $x$-axis by the factor $\sqrt{\rho_{\text {iso }} \kappa_{x x}}$. The different scaling factor along the $y$-axis is irrelevant, because there are no features (neither contacts nor boundaries) except on the $x$-axis. So the affine transformation (12a), (12b), and (12c) effectively just scales the entire geometry by a single factor, and this does not change ratios of voltages on (or currents through) the contacts or ratios of resistances between the contacts. In the end the absolute values of resistances of the transformed device indeed change due to $\rho_{\text {iso }}$ in $(7 \mathrm{~d})$. This shows a clear difference between Hall effect and piezoresistance effect: for the Hall effect we can transform devices onto the upper half plane with conformal transformation and preserve the Hall output voltage, whereas for the piezoresistance effect any direct conformal transformation onto the upper half plane would make the piezoresistive output voltage vanish. The correct procedure for a piezoresistive potential problem is to apply the affine transformation (12a), (12b), and (12c) prior to conformal transformation(s): the affine transformation establishes the validity of Laplace's equation and then it is allowed to go on with conformal transformations. Figure 5 shows numerical simulations of such a device in the upper half plane with piezoresistive coefficients of low n-doped silicon. The input voltage is forced by an external voltage supply between the outer contacts. Then the change of output voltage at the center contact caused by mechanical stress is close to zero (except for numerical inaccuracies) while the input resistance changes with stress according to $\rho_{\text {iso }}$ in (7d). This geometry has also some practical importance, because it is similar to vertical Hall devices $[39,40]$, where all contacts are on the top surface of a rectangular Hall region. In the early days this Hall region was the entire wafer and therefore it was close to infinite. The offset voltage of such a device can drift over lifetime due to drift of mechanical stress on the chip. With the above discussion we can say that for large Hall tubs this drift will get very small and for small Hall tubs it is caused by the isolating boundary at the sidewalls and bottom of the tub but not by the top surface. This was already stated in [41] based on the same theory using affine and conformal transformations. It was also experimentally observed that the offset of vertical Hall devices is more stable than the offset of conventional horizontal Hall plates [42].

\section{Devices with Mirror Symmetries to $x$ - and $y$-Axes and Contacts Not Lying on the $x$ - and $y$-Axes, at Zero Shear Stress $\sigma_{x y}=0$}

In standard microelectronic packages the main part of the chip (except its perimeter) is under pure inplane biaxial stress in the order of $100 \mathrm{MPa}$ [22]. Hence, this case is most relevant in practice. Then it holds for a (100)-silicon chip with (7a), (7d)

$$
\begin{aligned}
\rho_{\text {iso }} & =\frac{\rho_{0}}{2} \sqrt{2+\left(\pi_{11}+\pi_{12}-\pi_{44}\right) \sigma_{x x}+\left(\pi_{11}+\pi_{12}+\pi_{44}\right) \sigma_{y y}} \sqrt{2+\left(\pi_{11}+\pi_{12}+\pi_{44}\right) \sigma_{x x}+\left(\pi_{11}+\pi_{12}-\pi_{44}\right) \sigma_{y y}}, \\
\rho_{\text {iso }} \kappa_{x x} & =\sqrt{\frac{2+\left(\pi_{11}+\pi_{12}-\pi_{44}\right) \sigma_{x x}+\left(\pi_{11}+\pi_{12}+\pi_{44}\right) \sigma_{y y}}{2+\left(\pi_{11}+\pi_{12}+\pi_{44}\right) \sigma_{x x}+\left(\pi_{11}+\pi_{12}-\pi_{44}\right) \sigma_{y y}}} .
\end{aligned}
$$



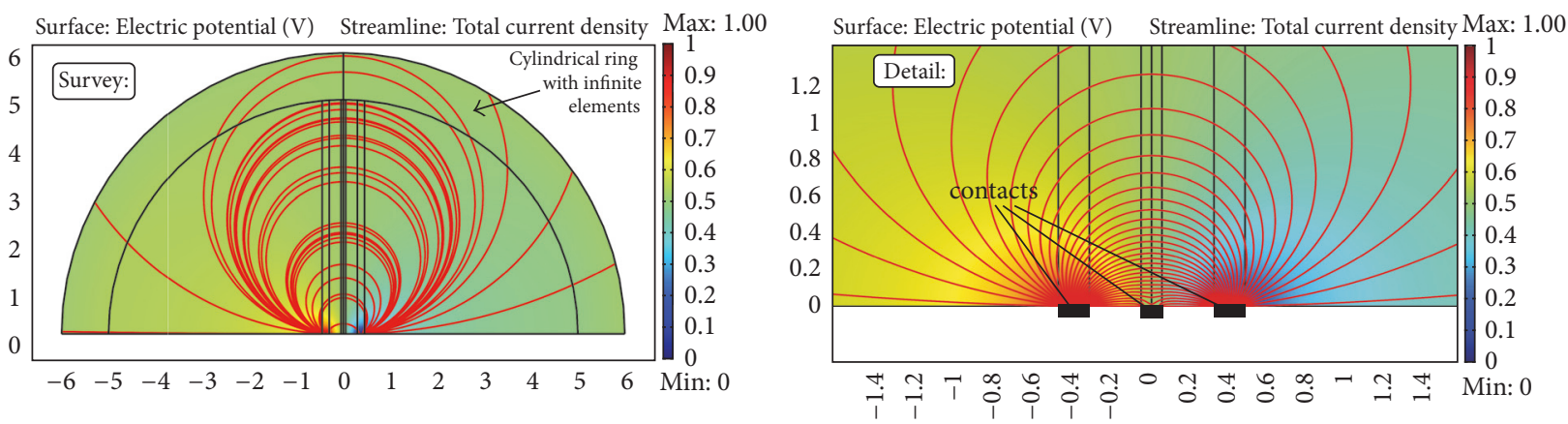

FIGURE 5: Finite element model of a resistive device with three contacts at the lower boundary (=x-axis). The plots were obtained by FEM simulations with COMSOL MULTIPHYSICS. They show the potential distribution and the current streamlines. The entire upper half plane is conductive with piezoresistance according to (5) and (6a)-(6c) with $\rho_{0}=1 \Omega \mathrm{m}$ and the piezoresistive coefficients for low n-doped silicon $\left(\pi_{11}=-1.022 / \mathrm{GPa}, \pi_{12}=0.534 / \mathrm{GPa}, \pi_{44}=-0.136 / \mathrm{GPa}\right)$. Pure strain is neglected. The outer contacts are $0.15 \mathrm{~m}$ long, the center contact is $0.1 \mathrm{~m}$ long, and the spacing between contacts is $0.25 \mathrm{~m}$. The thickness into the drawing plane is $1 \mathrm{~m}$. The left contact is forced to $1 \mathrm{~V}$, the right contact is forced to $0 \mathrm{~V}$, and the center contact is floating (i.e., no current flows in or out). The infinite boundary is modeled with infinite elements. The mesh has 1.4 million elements. At zero mechanical stress the center contact is at $0.5 \mathrm{~V}$ and the input resistance is $1.88262 \Omega$. At $\sigma_{x x}=100 \mathrm{MPa}$ the output voltage increases $57 \mathrm{nV}$ and the input resistance decreases $-2.443 \%$. At $\sigma_{y y}=100 \mathrm{MPa}$ the output voltage decreases $-56 \mathrm{nV}$ and the input resistance decreases $-2.441 \%$. At $\sigma_{x y}=100 \mathrm{MPa}$ the output voltage decreases $-2.5 \mu \mathrm{V}$ and the input resistance decreases $-1.218 \%$. All these resistance changes deviate less than $10 \mathrm{ppm}$ from theory (12a)-(12c). The changes in output voltage should be zero according to theory and the small deviations are probably due to insufficiently fine mesh.

For very large stress difference $\sigma_{x x}-\sigma_{y y}$ in the GPa-range the terms under the square-roots in (17a), (17b) become negative, and either $\rho_{x x}$ or $\rho_{y y}$ reverse sign (see (6a), (6b)), which is impossible for passive devices $(\vec{E} \cdot \vec{J}>0)$; there the firstorder piezoresistive theory breaks down.

4.1. Rectangular Devices with Four Large Contacts. With $(6 a)-(6 c),(12 a)$, and $(12 b)$ and with the results of $[10,11]$ we can immediately give the equivalent resistor circuit (ERC) and its dependence on stress for rectangular devices shown in Figure 6. We summarize these findings in (18a) $-(18 \mathrm{~m})$ :

$$
\begin{aligned}
\bar{R}_{H f} & =\frac{4 R_{\mathrm{sh}}}{4 \lambda_{x}+\lambda_{f}-\lambda_{p}}, \\
\bar{R}_{H p} & =\frac{4 R_{\mathrm{sh}}}{4 \lambda_{x}-\lambda_{f}+\lambda_{p}}, \\
\bar{R}_{D} & =\frac{2 R_{\mathrm{sh}}}{\lambda_{f}+\lambda_{p}-4 \lambda_{x}}, \\
\lambda_{f} & =\frac{K^{\prime}((1-f) /(1+f))}{K((1-f) /(1+f))} \\
\lambda_{p} & =\frac{K^{\prime}(2 \sqrt{p} /(1+p))}{K(2 \sqrt{p} /(1+p))} \\
4 \lambda_{x} & =\frac{K^{\prime}(((1-f) /(1+f))(2 \sqrt{p} /(1+p)))}{K(((1-f) /(1+f))(2 \sqrt{p} /(1+p)))}, \\
\frac{1-f}{1+f} & =\sqrt{\frac{1-k}{1+k} \frac{1+k w_{3}}{1-k w_{3}}},
\end{aligned}
$$

$$
\begin{aligned}
\frac{2 \sqrt{p}}{1+p} & =\sqrt{\frac{1+k}{2} \frac{1+w_{3}}{1+k w_{3}}}, \\
w_{3} & =\operatorname{sn}\left(K(k)\left(\frac{2 s_{0}}{\ell_{0}}-1\right), k\right), \\
k & =\sqrt{L\left(\frac{2 W}{\ell}\right)}, \\
W & =W_{0} \sqrt{\rho_{\text {iso }} \kappa_{x x}} \\
\ell & =\frac{\ell_{0}}{\sqrt{\rho_{\text {iso }} \kappa_{x x}}}, \\
R_{\text {sh }} & =\frac{\rho_{\text {iso }}}{t_{d}} .
\end{aligned}
$$

The parameters $\ell_{0}, W_{0}$, and $s_{0}$ are length, width, and spacing between contacts, respectively, of the device at zero stress (see Figure 6). They undergo the affine transformation (12a) $\ell_{0} \rightarrow \ell$ and $W_{0} \rightarrow W$ to account for the mechanical stress. The ratio $s_{0} / \ell_{0}=s / \ell$ is independent of stress, because both lengths are along $x$-direction and the common scaling factor of the affine transformation cancels out. $t_{d}$ is the constant thickness of the device. $R_{\mathrm{sh}}$ is the sheet resistance of the equivalent device with isotropic resistivity $\rho_{\text {iso }}$ after the affine transformation. $k \in[0,1], w_{3} \in[-1,1], f \in[0,1]$, and $p \in[0,1]$ are dimensionless parameters which appeared in the sequence of conformal transformations in $[10,11]$. In (18i) the Jacobi sine-amplitude function is used. It is defined by $x=$ $\operatorname{sn}(F(x, y), y)$. Equations (18a)-(18f) are given in $(14 \mathrm{a}-\mathrm{c})$ and (A11-13) in [10]; (18g)-(18j) are equivalent to $(8,9,14)$ in [11]. The parameters $\lambda_{f}, \lambda_{p}, \lambda_{x}$ depend on mechanical stress; if we refer to their values at zero stress, we add a zero in the indices: $\lambda_{f 0}, \lambda_{p 0}$, and $\lambda_{x 0}$. The ERC in Figure 6 and (18a)-(18m) is not 


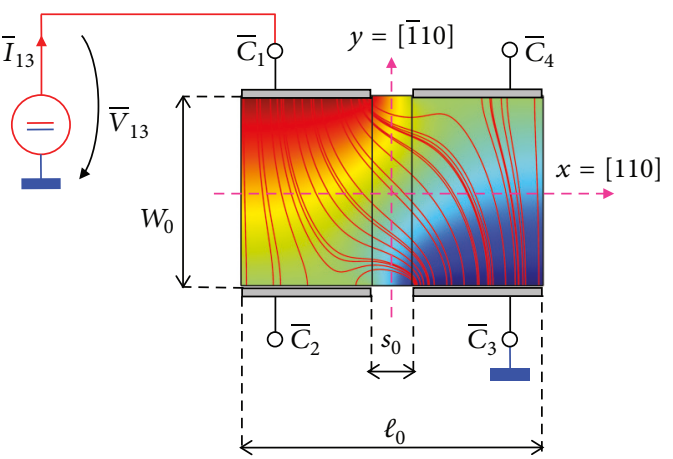

(a)

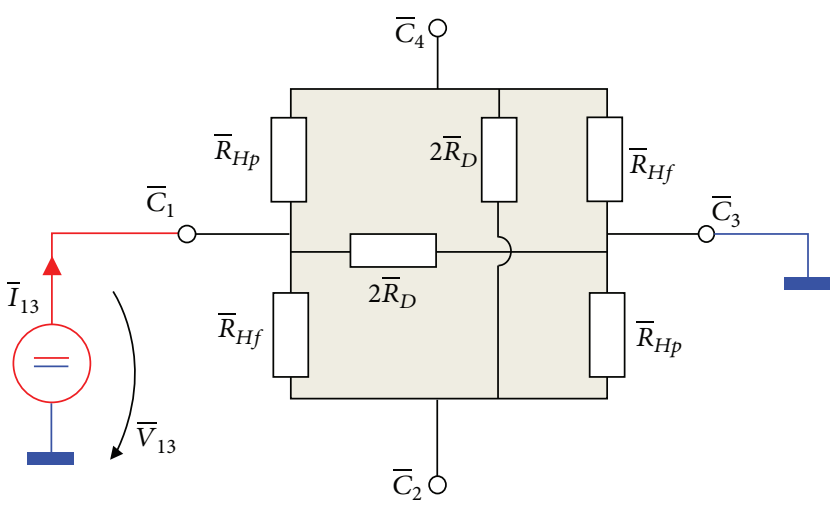

(b)

FIGURE 6: Rectangular $\sigma_{x x}-\sigma_{y y}$ stress sensor in (100)-silicon with two perpendicular mirror-symmetry axes $x=[110], y=[\overline{1} 10]$. No magnetic field is applied. An input voltage is forced between contacts $\bar{C}_{1}-\bar{C}_{3}$ and an output voltage is tapped between $\bar{C}_{2}-\bar{C}_{4}$. All contacts have the same size $\left(\ell_{0}-s_{0}\right) / 2$. (a) shows the top view of the potential distribution and the current streamlines in the plane device. (b) shows the equivalent resistor circuit (ERC).

an approximation; they are valid in a strict sense even for large mechanical stress and arbitrary size of contacts as long as we stay in the linear electrostatic and piezoresistive theory. With the ERC one can compute the input resistances, the ratios of output over input voltages, and the transimpedances of Halland van der Pauw-operating modes (all at zero magnetic field). For the Hall-operating mode it holds that

$$
\begin{aligned}
& \frac{\left.\left.\bar{V}_{13}\right|_{\bar{I}_{13}}\right|_{\bar{I}_{2}=\bar{I}_{4}=0}}{}=\bar{R}_{13}=\left.\frac{\bar{V}_{24}}{\bar{I}_{24}}\right|_{\bar{I}_{1}=\bar{I}_{3}=0}=\bar{R}_{24} \\
&=2 \bar{R}_{D} \frac{\bar{R}_{H f} \bar{R}_{H p}+\bar{R}_{D} \bar{R}_{H f}+\bar{R}_{D} \bar{R}_{H p}}{\left(\bar{R}_{H f}+2 \bar{R}_{D}\right)\left(\bar{R}_{H p}+2 \bar{R}_{D}\right)} \\
&=R_{\mathrm{sh}}\left(\frac{1}{\lambda_{p}}+\frac{1}{\lambda_{f}}\right), \\
&\left.\bar{V}_{24}\right|_{\bar{V}_{13}=\bar{I}_{4}=0}=\left.\frac{\bar{V}_{13}}{\bar{V}_{24}}\right|_{\bar{I}_{1}=\bar{I}_{3}=0} \\
&=\bar{R}_{D} \overline{\bar{R}}_{H f} \bar{R}_{H p}+\bar{R}_{D} \bar{R}_{H f}+\bar{R}_{D} \bar{R}_{H p} \\
&= \frac{\lambda_{f}-\lambda_{p}}{\lambda_{f}+\lambda_{p}}, \\
&\left.\bar{V}_{24}\right|_{\bar{I}_{2}=\bar{I}_{4}=0}=\left.\frac{\bar{V}_{13}}{\bar{I}_{24}}\right|_{\bar{I}_{1}=\bar{I}_{3}=0} \\
&=2 \bar{R}_{D}^{2} \frac{\bar{R}_{H p}-\bar{R}_{H f}}{\left(\bar{R}_{H f}+2 \bar{R}_{D}\right)\left(\bar{R}_{H p}+2 \bar{R}_{D}\right)} \\
&=R_{\mathrm{sh}}\left(\frac{1}{\lambda_{p}}-\frac{1}{\lambda_{f}}\right) .
\end{aligned}
$$

For the van der Pauw-operating mode it holds that

$$
\begin{aligned}
& \left.\frac{\bar{V}_{43}}{\bar{I}_{43}}\right|_{\bar{I}_{1}=\bar{I}_{2}=0}=\frac{\bar{R}_{H f}}{2} \frac{\bar{R}_{H f} \bar{R}_{H p}+2 \bar{R}_{D}\left(\bar{R}_{H f}+2 \bar{R}_{H p}\right)}{\left(\bar{R}_{H f}+2 \bar{R}_{D}\right)\left(\bar{R}_{H f}+\bar{R}_{H p}\right)} \\
& =R_{\mathrm{sh}}\left(\frac{1}{\lambda_{f}}+\frac{1}{4 \lambda_{x}}\right) \text {, } \\
& \left.\frac{\bar{V}_{23}}{\bar{I}_{23}}\right|_{\bar{I}_{1}=\bar{I}_{4}=0}=\frac{\bar{R}_{H p}}{2} \frac{\bar{R}_{H f} \bar{R}_{H p}+2 \bar{R}_{D}\left(2 \bar{R}_{H f}+\bar{R}_{H p}\right)}{\left(\bar{R}_{H p}+2 \bar{R}_{D}\right)\left(\bar{R}_{H f}+\bar{R}_{H p}\right)} \\
& =R_{\mathrm{sh}}\left(\frac{1}{\lambda_{p}}+\frac{1}{4 \lambda_{x}}\right) \text {, } \\
& \left.\frac{\bar{V}_{43}}{\bar{I}_{12}}\right|_{\bar{I}_{4}=\bar{I}_{3}=0}=\frac{\bar{R}_{H f}^{2}}{2} \frac{2 \bar{R}_{D}-\bar{R}_{H p}}{\left(\bar{R}_{H f}+2 \bar{R}_{D}\right)\left(\bar{R}_{H f}+\bar{R}_{H p}\right)} \\
& =R_{\mathrm{sh}}\left(\frac{1}{\lambda_{f}}-\frac{1}{4 \lambda_{x}}\right) \text {, } \\
& \left.\frac{\bar{V}_{43}}{\bar{V}_{12}}\right|_{\bar{I}_{4}=\bar{I}_{3}=0}=\bar{R}_{H f} \frac{2 \bar{R}_{D}-\bar{R}_{H p}}{\bar{R}_{H f} \bar{R}_{H p}+2 \bar{R}_{D}\left(\bar{R}_{H f}+2 \bar{R}_{H p}\right)} \\
& =\frac{4 \lambda_{x}-\lambda_{f}}{4 \lambda_{x}+\lambda_{f}}, \\
& \left.\frac{\bar{V}_{23}}{\bar{I}_{14}}\right|_{\bar{I}_{2}=\bar{I}_{3}=0}=\frac{\bar{R}_{H p}^{2}}{2} \frac{2 \bar{R}_{D}-\bar{R}_{H f}}{\left(\bar{R}_{H p}+2 \bar{R}_{D}\right)\left(\bar{R}_{H f}+\bar{R}_{H p}\right)} \\
& =R_{\mathrm{sh}}\left(\frac{1}{\lambda_{p}}-\frac{1}{4 \lambda_{x}}\right) \text {, } \\
& \left.\frac{\bar{V}_{23}}{\bar{V}_{14}}\right|_{\bar{I}_{2}=\bar{I}_{3}=0}=\bar{R}_{H p} \frac{2 \bar{R}_{D}-\bar{R}_{H f}}{\bar{R}_{H f} \bar{R}_{H p}+2 \bar{R}_{D}\left(\bar{R}_{H p}+2 \bar{R}_{H f}\right)} \\
& =\frac{4 \lambda_{x}-\lambda_{p}}{4 \lambda_{x}+\lambda_{p}}
\end{aligned}
$$




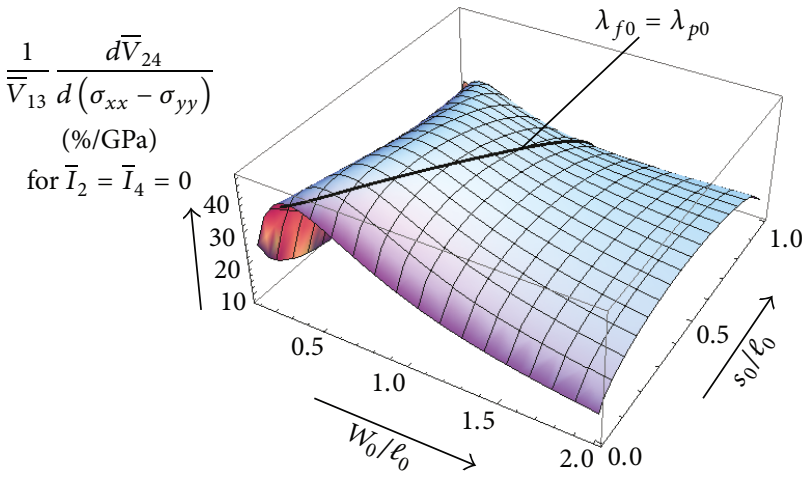

(a)

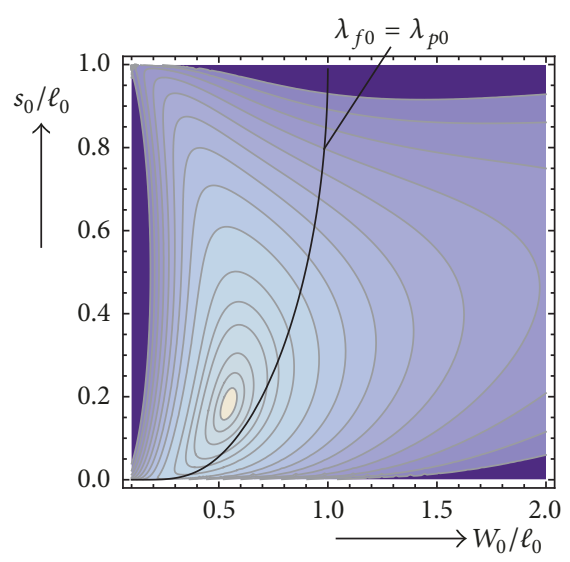

(b)

FIGURE 7: Sensitivity of output voltage $\bar{V}_{24}$ versus mechanical stress $\sigma_{x x}-\sigma_{y y}$ (normalized to supply voltage $\bar{V}_{13}$ ) for a device of Figure 6 operated in the same way as in Figure 6. All other components of the stress tensor vanish, as well as $\sigma_{x x}+\sigma_{y y}=0$. (a) is a 3D-plot and (b) shows the contour lines thereof. In (a) the steep slopes of the surface near $W_{0} / \ell_{0}<0.1$ and $s_{0} / \ell_{0}<0.01$ and $s_{0} / \ell_{0}>0.99$ are only sketched due to numerical problems. The plots are graphical representations of $(18 \mathrm{a})-(18 \mathrm{~m})$ in the limit of small stress and with the piezoresistive coefficient $\pi_{44}=1.381 / \mathrm{GPa}$ for low $\mathrm{p}$-doped silicon. Largest stress sensitivities are obtained for aspect ratios $W_{0} / \ell_{0} \approx 0.55$. The dependence on contact size is small, but $s_{0} / \ell_{0} \approx 0.18$ tends to be best. The black curve denotes offset-free devices with $\lambda_{f 0}=\lambda_{p 0}$, where $\bar{V}_{24}=0$ at zero stress. The maximum of the surface does not lie on this curve. The contours in (b) are for 46.75, 46.5, 46, 45, 44, 42.5, 40, .., 22.5, $20 \% / \mathrm{GPa}$.

whereby $\bar{I}_{m n}$ is the current which enters the device through contact $\bar{C}_{m}$ and leaves through contact $\bar{C}_{n}$ and $\bar{V}_{m n}$ is the voltage across both contacts $\bar{V}_{m n}=\phi\left(\bar{C}_{m}\right)-\phi\left(\bar{C}_{n}\right)$.

Only for carefully chosen contact spacing $s_{0}$ does the output voltage $\bar{V}_{24}$ vanish at zero stress in the Hall-operating mode $\bar{I}_{2}=\bar{I}_{4}=0$ and $\bar{I}_{3}=-\bar{I}_{1}$ : we get these offset-free devices with $\bar{R}_{H f}=\bar{R}_{H p}$ at zero stress (see (19b)) and this means $\lambda_{f 0}=\lambda_{p 0}$, from which follows the contact spacing (see [12])

$$
\begin{array}{r}
\frac{s_{0}}{\ell_{0}}=\frac{1}{2}\left(1+\frac{F\left(1 / k_{0}-8 /\left(1+4 k_{0}-k_{0}^{2}\right), k_{0}\right)}{K\left(k_{0}\right)}\right) \\
\text { with } k_{0}=\sqrt{L\left(2 \frac{W_{0}}{\ell_{0}}\right)} \text { for } \frac{W_{0}}{\ell_{0}} \leq 1
\end{array}
$$

Offset-free devices are only possible if the width $W_{0}$ is smaller or equal to the length $\ell_{0}$. Figure 7 shows the sensitivity of the output voltage $\bar{V}_{24}$ in Hall-operating mode with respect to small changes in stress $\sigma_{x x}-\sigma_{y y}$ at fixed supply voltage $\bar{V}_{13}$ for devices of Figure 6 and operated according to Figure 6. There the stress sensitivity function has a flat maximum of $46.839 \% / \mathrm{GPa}$ at $W_{0} / \ell_{0} \cong 0.5444, s_{0} / \ell_{0} \cong 0.1809$. At zero stress the number of squares between contacts $\bar{C}_{1}-\bar{C}_{3}$ or between contacts $\bar{C}_{2}-\bar{C}_{4}$ is 1.3386 (with $\lambda_{f 0} \cong 1.7538, \lambda_{p 0} \cong$ 1.3013). The output voltage at zero stress is $14.81 \%$ of the supply voltage; this device is not offset-free $\left(\lambda_{f 0} \neq \lambda_{p 0}\right)$. All these values were checked with a finite element simulation. If we restrict the search to offset-free devices the maximum stress sensitivity is only $2.5 \%$ smaller, namely, $45.674 \% / \mathrm{GPa}$ at $W_{0} / \ell_{0} \cong 0.6220$ and $s_{0} / \ell_{0} \cong 0.1315$ (with $\lambda_{f 0}=\lambda_{p 0} \cong$
1.5733, see Figure 8); then the resistance between even or odd contacts has 1.2712 squares. The value of both maxima is computed for $\pi_{44}=1.381 / \mathrm{GPa}$ of low $\mathrm{p}$-doped silicon; in case of other materials of a cubic crystal class the stress sensitivity scales linearly with $\pi_{44}$ [43].

The theory was verified by finite element simulations at small and large stress (Figure 9). The device had the geometric parameters $W_{0}=0.62207 \mathrm{~m}, \ell_{0}=t_{d}=1 \mathrm{~m}$, and $s_{0}=0.13149 \mathrm{~m}$. For the conductance a linear material law was used according to (7a)-(7d) with $\rho_{0}=1 \Omega \mathrm{m}$ and $\pi_{11}=6.6 \times 10^{-11} / \mathrm{Pa}, \pi_{12}=-1.1 \times 10^{-11} / \mathrm{Pa}$, and $\pi_{44}=$ $138.1 \times 10^{-11} / \mathrm{Pa}$. The two-dimensional simulation had a fine mesh of 1.76 million elements. It used Lagrange multiplier for improved accuracy of currents into the contacts. Table 1 compares the results of this numerical simulation with results of the analytical theory comprising (17a), (17b), (18a)-(18m), (19a), and (19b). Even up to very large stress levels FEM and the theory agrees better than $0.1 \%$.

4.2. Rectangular Devices with Four Point-Sized Contacts. If the contacts of the device in Figure 6 get very small we have $s_{0} \rightarrow \ell_{0}-2 d s_{0}$, which we plug into (18i) and develop that into a series in small $d s_{0}$.

$$
w_{3} \longrightarrow 1-8\left(1-k^{2}\right) K^{2}(k)\left(\frac{d s_{0}}{\ell_{0}}\right)^{2}
$$

Inserting this into (18g), (18h), and (18d)-(18f) gives

$$
\lambda_{f} \rightarrow \frac{-\pi}{2} \frac{1}{\ln \left(\sqrt{k} K(k) d s_{0} / \ell_{0}\right)}
$$




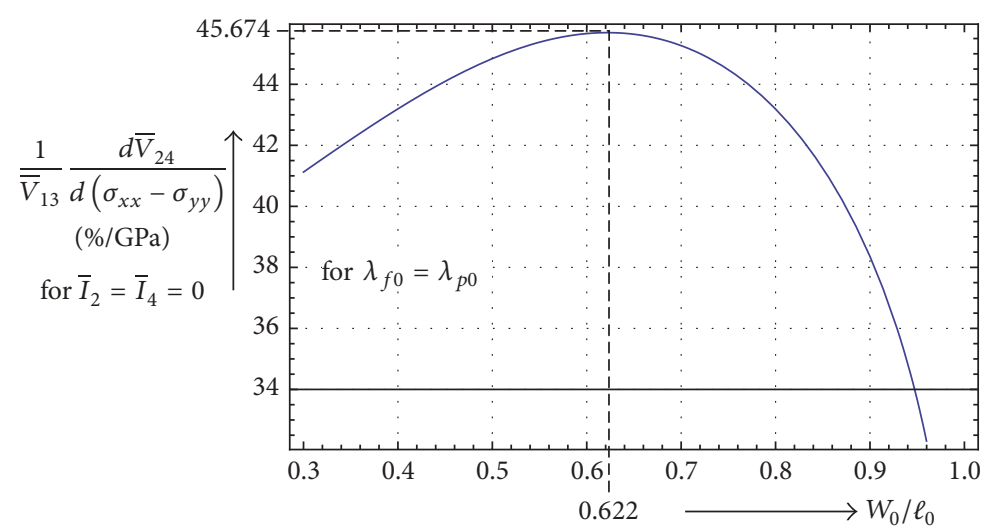

Figure 8: Sensitivity of output voltage $\bar{V}_{24}$ versus mechanical stress $\sigma_{x x}-\sigma_{y y}$ (normalized to supply voltage $\bar{V}_{13}$ ) for an offset-free device of Figure 6 operated in the same way as in Figure 6. All other components of the stress tensor vanish, as well as $\sigma_{x x}+\sigma_{y y}=0$. The plot is a graphical representation of $(18 \mathrm{a})-(18 \mathrm{~m})$ in the limit of small stress and with the piezoresistive coefficient $\pi_{44}=1.381 / \mathrm{GPa}$ for low $\mathrm{p}$-doped silicon. Largest stress sensitivity of $45.674 \% / \mathrm{GPa}$ is obtained for aspect ratios $W_{0} / \ell_{0} \approx 0.622$. There $s_{0} / \ell_{0} \cong 0.1315$ satisfies $(20)$.

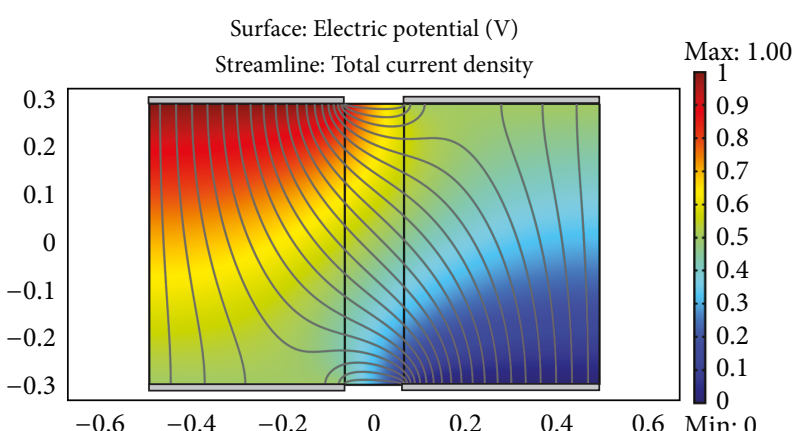

(a)

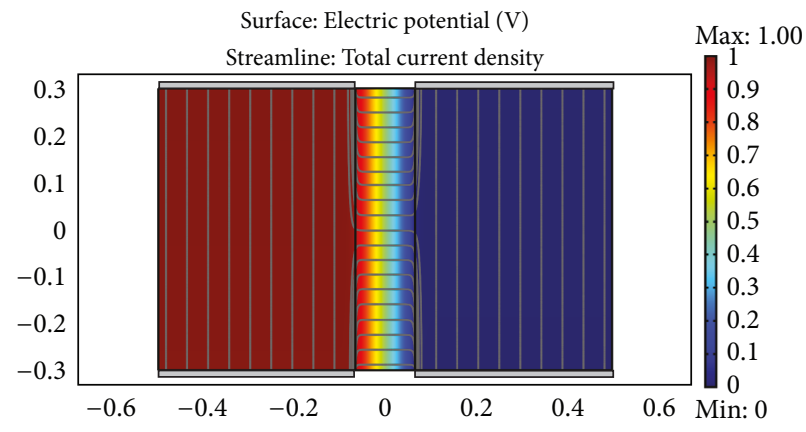

(b)

FIGURE 9: Rectangular device from Figure 6 with $W_{0}=0.62207 \mathrm{~m}, s_{0}=0.13149 \mathrm{~m}$ for comparison of the analytical theory with a finite element simulation with COMSOL MULTIPHYSICS. The plots show the potential and current streamlines. (a) The left plot is for small mechanical stress difference $\sigma_{x x}-\sigma_{y y}$ (third line of Table 1); (b) the right plot is for large mechanical stress difference (last line of Table 1).

$$
\begin{gathered}
\lambda_{p} \longrightarrow 4 \lambda_{x} \longrightarrow \\
\frac{\pi}{2} \frac{1}{\ln 2-\ln \left((1-k) K(k) d s_{0} / \ell_{0}\right)}
\end{gathered}
$$

Inserting this into (19a), (19b) gives

$$
\begin{aligned}
& \left.\frac{\bar{V}_{13}}{\bar{I}_{13}}\right|_{\bar{I}_{2}=\bar{I}_{4}=0} \longrightarrow \frac{2}{\pi} \\
& \cdot R_{\mathrm{sh}}\left(\ln 2-\ln \left(\sqrt{k}(1-k) K^{2}(k)\left(\frac{d s_{0}}{\ell_{0}}\right)^{2}\right)\right) \\
& \frac{\bar{V}_{24}}{\bar{V}_{13}} \longrightarrow \frac{\ln (2 \sqrt{k})-\ln (1-k)}{\ln 2-\ln \left(\sqrt{k}(1-k) K^{2}(k)\left(d s_{0} / \ell_{0}\right)^{2}\right)}
\end{aligned}
$$

$$
\frac{\bar{V}_{24}}{\bar{I}_{13}} \longrightarrow \frac{2}{\pi} R_{\mathrm{sh}} \ln \left(\frac{2 \sqrt{k}}{1-k}\right)
$$

(22a)-(22c) hold for small contacts and arbitrary stress. $k$ changes with stress according to (18j). For small stress we write $k=k_{0}+d k$, where $k_{0}$ is the value at zero stress and $d k$ is the small stress variation of $k$. For point-sized contacts $s_{0} / \ell_{0} \rightarrow 1$ and then the only offset-free geometry with $\bar{V}_{24}=$ 0 at zero stress is $k_{0}=3-2 \sqrt{2}$, which gives $W_{0} / \ell_{0}=1$ (cf. (20)). From (18k), (18l), and (17b) we get for small stress

$$
\begin{aligned}
& \frac{W}{\ell}=\frac{\rho_{\text {iso }} \kappa_{x x} W_{0}}{\ell_{0}} \longrightarrow \\
& 1-\frac{\pi_{44}\left(\sigma_{x x}-\sigma_{y y}\right)}{2}
\end{aligned}
$$


TABLE 1: Mechanical stress sensitivity of rectangular device from Figure 9: comparison of numerical simulation (FEM) versus analytical theory. $\pi_{11}=6.6 \times 10^{-11} / \mathrm{Pa}, \pi_{12}=-1.1 \times 10^{-11} / \mathrm{Pa}, \pi_{44}=138.1 \times 10^{-11} / \mathrm{Pa}$. The small residual output voltage of the FEM in the first line is probably due to insufficiently fine mesh. In the last line the stress difference $\sigma_{x x}-\sigma_{y y}$ is so large that $\rho_{\text {iso }} / \rho_{0}$ gets nearly zero. Then $\lambda_{f}$ gets large ( 48.9), $\lambda_{p}$ gets small ( 0.13), $\rho_{y y} / \rho_{0}$ gets small ( 0.0017), and the output voltage approaches the supply voltage. For even larger stress difference the first-order piezoresistive theories $(6 \mathrm{a})$ and $(6 \mathrm{~b})$ break down and also the numerical simulation gives meaningless results.

\begin{tabular}{lcccccc}
\hline$\sigma_{x x}[\mathrm{MPa}]$ & $\sigma_{y y}[\mathrm{MPa}]$ & $\rho_{\text {iso }} / \rho_{0}$ & $\bar{V}_{24} / \bar{V}_{13} \mathrm{FEM}$ & $\bar{V}_{24} / \bar{V}_{13}$ theory & $\bar{V}_{13} / \bar{I}_{13}[\Omega]$ FEM & $\bar{V}_{13} / \bar{I}_{13}[\Omega]$ theory \\
\hline 0 & 0 & 1.00000 & -0.000229 & $-4 \times 10^{-8}$ & 1.27104 & 1.27140 \\
10 & 0 & 1.00025 & 0.004341 & 0.004570 & 1.26859 & 1.26895 \\
0 & 10 & 1.00025 & -0.004792 & -0.004563 & 1.27416 & 1.27174 \\
10 & 10 & 1.00055 & -0.000229 & $-4 \times 10^{-8}$ & 1.30872 & 1.27210 \\
1000 & 900 & 1.04998 & 0.043525 & 0.043750 & 1.23156 & 1.30910 \\
-1000 & -900 & 0.94523 & -0.048074 & -0.047841 & 0.45175 & 0.45216 \\
856 & -600 & 0.05801 & 0.994747 & 0.994751 & & \\
\hline
\end{tabular}

Plugging this into (18j) and developing for small stress gives

$$
\begin{aligned}
k & \longrightarrow \\
k_{0} & +d k \\
& =k_{0}-\left(\frac{2}{\pi}\right) k_{0}\left(k_{0}^{2}-1\right) K^{2}\left(k_{0}\right) \pi_{44}\left(\sigma_{x x}-\sigma_{y y}\right)
\end{aligned}
$$

In (23b) we used the differentiation rule for the modular lambda function

$$
\frac{d L(y)}{d y}=\left(\frac{4}{\pi}\right) L(y)(L(y)-1) K^{2}(\sqrt{L(y)})
$$

which can be proven by

$$
\begin{aligned}
\frac{d L(y)}{d y} & =\frac{1}{d y / d L}=\left(\frac{d\left(K^{\prime}(k) / K(k)\right)}{d k^{2}}\right)^{-1} \\
& =\frac{-2\left(1-k^{2}\right) k^{2} K^{2}(k)}{E(k) K^{\prime}(k)-K(k) K^{\prime}(k)+E^{\prime}(k) K(k)}
\end{aligned}
$$

with the substitution $y=K^{\prime}(k) / K(k)$ and $L(y)=k^{2}$. The denominator at the right hand side of $(24 \mathrm{~b})$ is equal to $\pi / 2$ according to Legendre's relation [44]. In (24b) $E(k)$ is the complete elliptic integral of the second kind, defined by $E(k)=\int_{0}^{\pi / 2} \sqrt{1-k^{2} \sin ^{2} \alpha} d \alpha$ and $E^{\prime}(k)=E\left(\sqrt{1-k^{2}}\right)$. Inserting (23b) into (22c) and developing for small stress gives

$$
\begin{aligned}
\frac{1}{\bar{I}_{13}} \frac{d \bar{V}_{24}}{d\left(\sigma_{x x}-\sigma_{y y}\right)} & \longrightarrow \\
\frac{128 \pi}{\Gamma^{4}(-1 / 4)} \pi_{44} R_{\mathrm{sh}} & \cong 0.696602 \times \pi_{44} R_{\mathrm{sh}} \\
& \cong 3.157251 \times \frac{\ln 2}{\pi} \pi_{44} \frac{\rho_{0}}{t_{d}}
\end{aligned}
$$

whereby $\Gamma(y)$ is the gamma function and we used $K(3-$ $2 \sqrt{2})=(2 \pi)^{3 / 2}(1+\sqrt{2}) / \Gamma^{2}(-1 / 4)[45] .(25 a)$ is identical with (16) in [46]. It was also checked by an FEM simulation. If the devices are not square but general rectangles with $W_{0} / \ell_{0} \neq 1$ they are not offset-free and the transconductance is

$$
\begin{aligned}
& \frac{\bar{V}_{24}}{\bar{I}_{13}} \longrightarrow \\
& \frac{2}{\pi} R_{\mathrm{sh}}\left\{\ln \frac{2 L_{0}^{1 / 4}}{1-\sqrt{L_{0}}}+\frac{1}{\pi} \frac{W_{0}}{\ell_{0}}\left(1+\sqrt{L_{0}}\right)^{2}\right. \\
& \left.\cdot\left(K\left(\sqrt{L_{0}}\right)\right)^{2} \pi_{44}\left(\sigma_{x x}-\sigma_{y y}\right)\right\} \\
& \text { with } L_{0}=L\left(\frac{2 W_{0}}{\ell_{0}}\right)
\end{aligned}
$$

A plot of (25b) reveals that square devices have smaller stress sensitivity $d \bar{V}_{24} / d\left(\sigma_{x x}-\sigma_{y y}\right)$ at given input current $\bar{I}_{13}$ than devices with large aspect ratio.

4.3. Circular Disk Shaped Devices with Four Large Contacts. Figure 10(a) shows a circular device with two pairs of contacts of different size: each contact covers an aperture angle of $2 \theta_{f}$ and $2 \theta_{p}$, respectively. Thus, its layout has two DoFs just like the device of Figure 6 . The device has two perpendicular mirror symmetries, namely, the $x$ - and $y$-axes. We assume that it is implemented in (100)-silicon with an alignment between the $(x, y)$-coordinate system and the crystal system like in Figure 1. Under a biaxial mechanical load along the symmetry axes the resistivity tensor becomes anisotropic $\left(\rho_{x x} \neq \rho_{y y}\right)$ but the offdiagonal elements vanish $\left(\rho_{x y}=0\right)$. As explained above we may apply the affine transformation (12a), which makes an ellipse in the $z^{\prime}$-plane out of the circle in the original $\underline{z}$-plane (see Figure $10(\bar{b})$ ). This device has the same potentials and currents at all contacts as the device in Figure 10(a), even though in Figure 10(b) the resistivity is isotropic and the potential is a solution of the Laplace equation, whereas in Figure 10(a) the resistivity is anisotropic and the potential is no solution of the Laplace equation. Thereby the stress $\sigma_{x x}$ acts along a line, where the contacts with angles $2 \theta_{f}$ are arranged. The stress $\sigma_{y y}$ acts along a line, where the contacts with angles $2 \theta_{p}$ are arranged. In a second step we use a conformal transformation $\underline{z^{\prime \prime}}=-j \sqrt{\rho_{\text {iso }} \kappa_{x x}} z^{\prime} / \sqrt{\left(\rho_{\text {iso }} \kappa_{x x}\right)^{2}-1}$ with $j$ being the imaginary unit, which rotates the device by $90^{\circ}$ in 


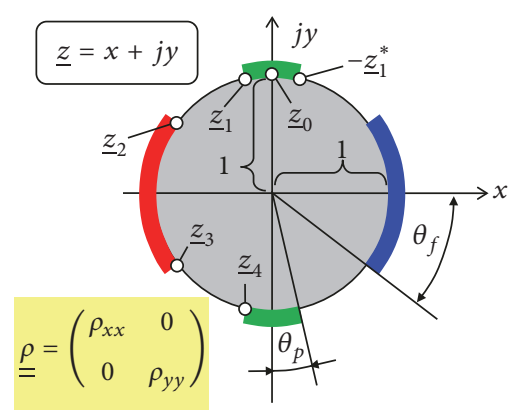

(a)

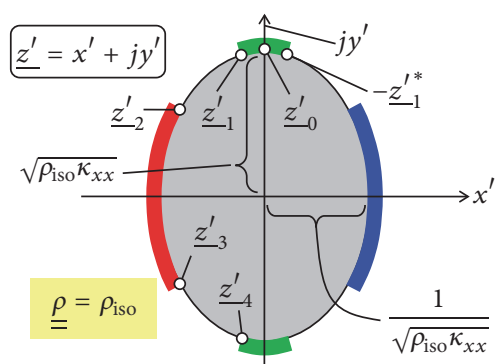

(b)

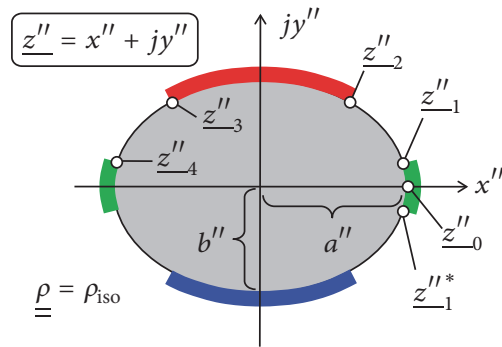

(c)

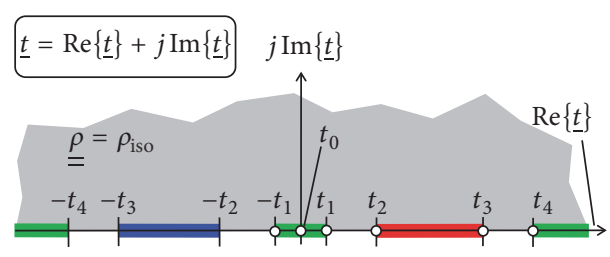

(d)

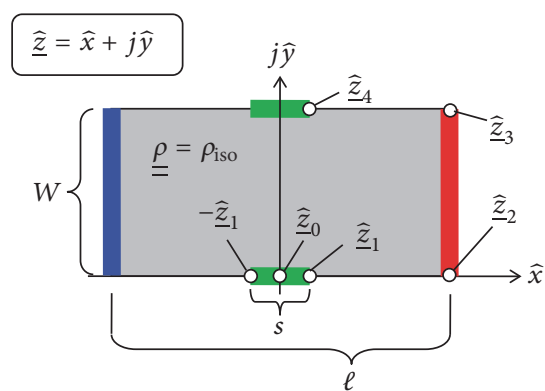

(e)

Figure 10: (a-d) Mapping a circular device with anisotropic conductivity by one affine and three conformal transformations onto an equivalent rectangular device with isotropic conductivity. (a) shows a circular disk shaped device with two pairs of contacts and two perpendicular mirror-symmetry axes $x$ and $y$. Its resistivity is anisotropic with the principal axes $x$ and $y$. This describes the action of biaxial load along $x$ - and $y$-axes on chips in (100)-silicon aligned like in Figure 1. The elliptical device in (b) with isotropic resistivity is equivalent to the device in (a). The shape in (b) is obtained by the affine transformation $\underline{z} \rightarrow \underline{z^{\prime}}$ (12a). The conformal transformation $\underline{z^{\prime}} \rightarrow \underline{z^{\prime \prime}}$ rotates the device clockwise by $90^{\circ}$ and scales it isotropically: $a^{\prime \prime}=\rho_{\text {iso }} \kappa_{x x} / \sqrt{\left(\rho_{\text {iso }} \kappa_{x x}\right)^{2}-1}$ and $b^{\prime \prime}=1 / \sqrt{\left(\rho_{\text {iso }} \kappa_{x x}\right)^{2}-1}$ with $a^{\prime \prime 2}-b^{\prime \prime 2}=1$. A second-conformal transformation $z^{\prime \prime} \rightarrow \underline{t}$ (26a) and (26b) maps the interior of the ellipse in (c) onto the upper half of the $t$-plane in (d). A third-conformal transformation $\underline{t} \rightarrow \underline{\widehat{z}}$ maps the upper half of the $t$-plane into the interior of the rectangle in (e). The contacts along the perimeter of the shapes relate to the contacts on the real $t$-axis. Corresponding points have equal indices. The sequence of points with indices $0,1,2,3$, and 4 is always counterclockwise while the conductive region is at the left hand side of this path. The asterisk means the conjugate complex.

clockwise direction and scales it isotropically with the factor $\sqrt{\rho_{\text {iso }} \kappa_{x x} /\left(\left(\rho_{\text {iso }} \kappa_{x x}\right)^{2}-1\right)}$ such that the foci of the ellipse are at $\underline{z^{\prime \prime}}= \pm 1$. A second-conformal transformation $\underline{z^{\prime \prime}} \rightarrow \underline{t}$ maps the interior of the ellipse onto the upper half of the $\underline{t}$-plane (Figure 10(d)), as explained in [48]. At the boundary of the elliptical region the mapping is

$$
\begin{aligned}
& \stackrel{z^{\prime \prime}}{=} \frac{\rho_{\mathrm{iso}} \kappa_{x x}}{\sqrt{\left(\rho_{\mathrm{iso}} \kappa_{x x}\right)^{2}-1}} \cos \left(\frac{\pi}{K(\kappa)} F\left(\frac{t}{\sqrt{1+t^{2}}}, \kappa\right)\right) \\
& \quad+\frac{j}{\sqrt{\left(\rho_{\mathrm{iso}} \kappa_{x x}\right)^{2}-1}} \sin \left(\frac{\pi}{K(\kappa)} F\left(\frac{t}{\sqrt{1+t^{2}}}, \kappa\right)\right), \\
& \kappa=\sqrt{L\left(\frac{1}{2 \pi} \ln \left(\frac{\rho_{\text {iso }} \kappa_{x x}+1}{\rho_{\text {iso }} \kappa_{x x}-1}\right)\right)} ;
\end{aligned}
$$

$\underline{z^{\prime \prime}}$ and $\underline{t}$ are complex numbers and $t=\operatorname{Re}\{\underline{t}\}$. (26a) and (26b) are only valid for $\rho_{\text {iso }} \kappa_{x x}>1$, which means $\pi_{44}\left(\sigma_{x x}-\sigma_{y y}\right)<0$. If we move on the $t$-axis from $-\infty$ over $t_{0}=0$ to $\infty$ this means a walk in the $z^{\prime \prime}$-plane in counterclockwise direction starting at $x^{\prime \prime}=-\rho_{\text {iso }} \kappa_{x x} / \sqrt{\left(\rho_{\text {iso }} \kappa_{x x}\right)^{2}-1}$ and moving through the lower $\underline{z}^{\prime \prime}$-plane over $x^{\prime \prime}=\underline{z}_{0}^{\prime \prime}=\rho_{\text {iso }} \kappa_{x x} / \sqrt{\left(\rho_{\text {iso }} \kappa_{x x}\right)^{2}-1}$ back to $x^{\prime \prime}=-\rho_{\text {iso }} \kappa_{x x} / \sqrt{\left(\rho_{\text {iso }} \kappa_{x x}\right)^{2}-1}$ through the upper $z^{\prime \prime}$ plane. Thus, positive real $t$ corresponds to the upper half of the edge of the ellipse. Then the end points of the contacts in the $\underline{t}$-plane are

$$
\begin{aligned}
\left(1-\kappa^{2}\right)^{-1 / 4} & \geq t_{1}=\operatorname{sc}\left(\frac{\theta_{p}}{\pi} K(\kappa), \kappa\right) \geq 0, \\
\left(1-\kappa^{2}\right)^{-1 / 4} & \geq t_{2}=\operatorname{sc}\left(\left(\frac{1}{2}-\frac{\theta_{f}}{\pi}\right) K(\kappa), \kappa\right) \geq t_{1}, \\
t_{3} & =\operatorname{sc}\left(\left(\frac{1}{2}+\frac{\theta_{f}}{\pi}\right) K(\kappa), \kappa\right) \\
& \geq\left(1-\kappa^{2}\right)^{-1 / 4}, \\
t_{4} & =\operatorname{sc}\left(\left(1-\frac{\theta_{p}}{\pi}\right) K(\kappa), \kappa\right) \geq t_{3}
\end{aligned}
$$



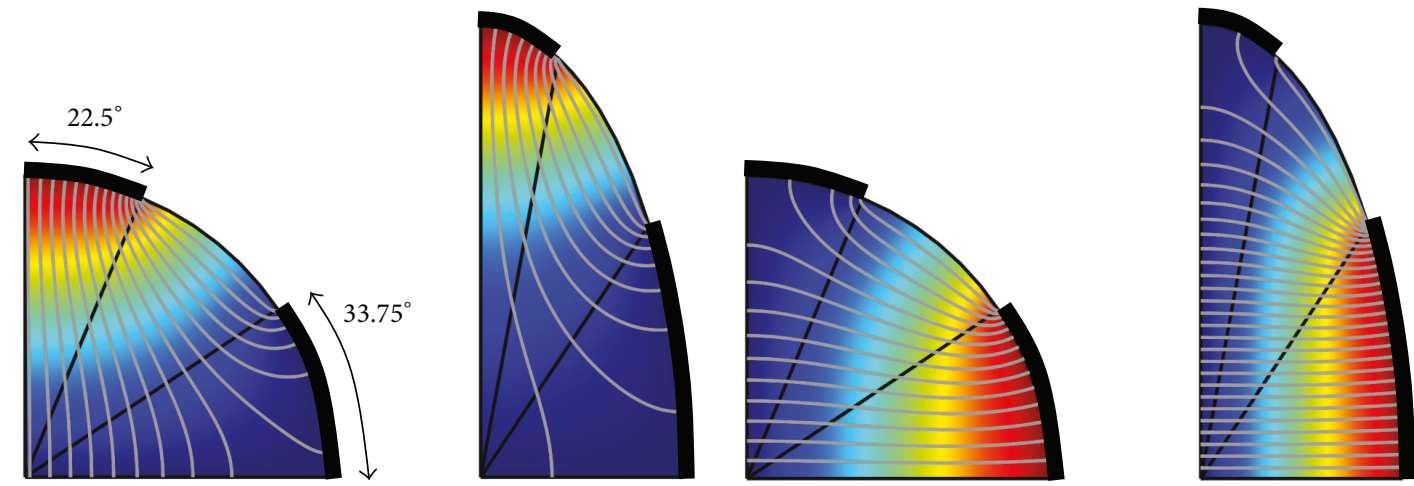

FIGURE 11: FEM-computation of input and output resistances of circular and elliptical devices with isotropic conductivity. Due to symmetry only a quarter of the real devices had to be modeled. The elliptical device is obtained from the circular one by stretching it times 1.5 in vertical direction and times $2 / 3$ in horizontal direction. Contacts are thick black lines on the perimeter. The color coding denotes the electric potential (red $=1 \mathrm{~V}$, blue $=0 \mathrm{~V})$ and the gray lines are current streamlines.

for $0<\theta_{f}+\theta_{p}<90^{\circ}$ and $\operatorname{sc}(y, \kappa)=\operatorname{sn}(y, \kappa) / \operatorname{cn}(y, \kappa)$, $\operatorname{sn}(y, \kappa)$, and $\operatorname{cn}(y, \kappa)$ being sine and cosine of the Jacobi amplitude. Next we map the interior of the rectangular device of Figure 10(e) from its $\underline{\underline{z}}$-plane onto the upper half of the $\underline{t}$ plane in Figure 10(d) via the Schwartz-Christoffel transformation $\underline{\underline{z}}=W F\left(\underline{t} / t_{2}, k\right) / K^{\prime}(k)$ with $\ell / W=2 K(k) / K^{\prime}(k)$. Thereby the center $\underline{\underline{z}}_{0}$ of the lower partial contact is mapped onto the origin in the $t$-plane. The device in Figure 10(d) is identical to the even symmetry device in [10]. Therefore the flush contacts at positions $\widehat{x}= \pm \ell / 2$ have the resistance $\lambda_{f} R_{\mathrm{sh}}$ with $R_{\mathrm{sh}}=\rho_{\mathrm{iso}} / t_{d}$ between them, and the resistance between the partial contacts is $\lambda_{p} R_{\mathrm{sh}}$ (see [10]). Applying the Schwartz-Christoffel transformation to $\widehat{\underline{z}}_{3}$ gives $F\left(t_{3} / t_{2}, k\right)=$ $K(k)+j K^{\prime}(k)$. However, this is identical to $F(1 / k, k)$. Therefore, it holds $k=t_{2} / t_{3}$, and with (27b) and (27c) we get

$$
k=\frac{\operatorname{sc}\left(\left(1 / 2-\theta_{f} / \pi\right) K(\kappa), \kappa\right)}{\operatorname{sc}\left(\left(1 / 2+\theta_{f} / \pi\right) K(\kappa), \kappa\right)}
$$

With the half-period addition theorem $\operatorname{sc}(z-K(\kappa), \kappa)=-(1-$ $\left.\kappa^{2}\right)^{-1 / 2} / \operatorname{sc}(z, \kappa)$ it follows that [49]

$$
k=\sqrt{1-\kappa^{2}} \operatorname{sc}^{2}\left(\left(\frac{1}{2}-\frac{\theta_{f}}{\pi}\right) K(\kappa), \kappa\right)
$$

Finally, we apply the Schwartz-Christoffel transformation to $\underline{\underline{z}}_{1}: s / 2=W F\left(t_{1} / t_{2}, k\right) / K^{\prime}(k)$. With (27a) and (27b) this gives

$$
\frac{s}{\ell}=\frac{1}{K(k)} F\left(\frac{\mathrm{sc}\left(\pi^{-1} \theta_{p} K(\kappa), \kappa\right)}{\operatorname{sc}\left(\left(1 / 2-\theta_{f} / \pi\right) K(\kappa), \kappa\right)}, k\right)
$$

With (28b) and (28c) and with $s / \ell=s_{0} / \ell_{0}$ we immediately know $w_{3}$ from (18i), and with (18d)-(18h) we can compute $\lambda_{f}, \lambda_{p}$, and $\lambda_{x}$. With these parameters we know all resistors in the ERC of the even symmetry device in Figure 10(a) (see [10]) and also of the odd symmetry device in Figure 12(a). With (19a)-(19i) we get all impedance, voltages, and transresistances in Hall- and van der Pauw-operating modes.
We checked (28b), (28c), and (18d)-(18h) with finite element calculations of the circular device in Figure 11 with $\theta_{p}=22.5^{\circ}$ and $\theta_{f}=33.75^{\circ}$ with isotropic conductivity. For zero stress we set $\rho_{\text {iso }} \kappa_{x x} \rightarrow 1$ and obtained $\lambda_{p}=$ 1.33951 and $\lambda_{f}=1.13789$ as input/output numbers of squares. Then we stretched the device in $x$ - and $y$-directions according to Figure $10(\mathrm{~b})$ with $\rho_{\text {iso }} \kappa_{x x} \rightarrow 2.25$ and obtained $k=0.0207218, s / \ell=0.0852258, \ell / W=0.596948, \lambda_{p}=$ 2.14874 , and $\lambda_{f}=0.594916$. Both times the results of FEM and analytical theory matched up to $0.04 \%$ for $\lambda_{p}$ and $\lambda_{f}$. Thereby, the FEM model had a mesh with 0.6 million elements per device.

For $\rho_{\text {iso }} \kappa_{x x}<1$ we must change the transformation between Figures $10(\mathrm{~b})$ and $10(\mathrm{c})$ to $\underline{z}^{\prime \prime}=\underline{z}^{\prime} \sqrt{\rho_{\text {iso }} \kappa_{x x}} /$ $\sqrt{1-\left(\rho_{\text {iso }} \kappa_{x x}\right)^{2}}$. This means to swap $\theta_{f}$ with $\theta_{p}$ in (28a)-(28c) and to replace $\rho_{\text {iso }} \kappa_{x x} \rightarrow\left(\rho_{\text {iso }} \kappa_{x x}\right)^{-1}$ in (26b). Since the new transformation $\underline{z^{\prime}} \rightarrow \underline{z^{\prime \prime}}$ does not rotate the device, $\theta_{f}$ contacts are now mapped onto the partial contacts of the rectangular device in Figure 10(e), so that we also have to swap $\lambda_{f}$ with $\lambda_{p}$.

We checked the correctness of (26b), (28b), (28c), and (18d)-(18i) with a series of finite element simulations given in Table 2.

With these foundations we can study the mechanical stress sensitivity of symmetric circular devices with arbitrary ratio of input/output resistances. Output voltages of devices from Figure 10 do not respond to $\sigma_{x x}-\sigma_{y y}$, because their contacts are on the axes of mirror-symmetry and even after the affine transformation (12a) they will stay on these symmetry axes so that their output signals remain zero. One can prove this by inserting $\lambda_{f}, \lambda_{p}$, and $\lambda_{x}$ from (18d) to (18f) into $(2 \mathrm{a}-\mathrm{c})$ of [10] to compute the equivalent resistor circuit (ERC) that applies to a device from Figure 10. This ERC is shown in Figure $2 b$ of [10] - it is different from the ERC of Figure 6 in this current work. For the measurement of $\sigma_{x x}-\sigma_{y y}$ we have to consider the complementary devices where contacts and isolating boundaries are swapped (see Figure 12(a)). In Figure 12(b) we checked the analytical theory against numerical simulations for various contact 
TABLE 2: Mechanical stress sensitivity of a circular device from Figure 10(a): comparison of numerical finite element simulation (FEM) versus analytical theory. $\lambda_{f} R_{\mathrm{sh}}$ is the resistance between the $\theta_{f}$-contacts in Figure 10 (a) and $\lambda_{p} R_{\mathrm{sh}}$ is the resistance between the $\theta_{p}$-contacts in Figure 10(a). The mesh of the FEM-simulation had 2.66 million elements and the model used Lagrange multiplier and the piezoresistivity matrix from (6a) to (6c) with $\pi_{11}=6.6 \times 10^{-11} / \mathrm{Pa}, \pi_{12}=-1.1 \times 10^{-11} / \mathrm{Pa}$, and $\pi_{44}=138.1 \times 10^{-11} / \mathrm{Pa}$. The theory used (18d), (18e), (18g), (18h), (18i), (28b), (28c), and (26b) for $\rho_{\text {iso }} \kappa_{x x}>1$. For $\rho_{\text {iso }} \kappa_{x x}<1$ we swapped $\theta_{f}$ with $\theta_{p}$ in (28b) and (28c) and $\lambda_{f}$ with $\lambda_{p}$, and we replaced $\rho_{\text {iso }} \kappa_{x x} \rightarrow\left(\rho_{\text {iso }} \kappa_{x x}\right)^{-1}$ in $(26 \mathrm{~b})$.

\begin{tabular}{lccccccccc}
\hline $\begin{array}{c}\theta_{f} \\
{\left[{ }^{\circ}\right]}\end{array}$ & $\begin{array}{c}\theta_{p} \\
{\left[{ }^{\circ}\right]}\end{array}$ & $\begin{array}{c}\sigma_{x x} \\
{[\mathrm{MPa}]}\end{array}$ & $\begin{array}{c}\sigma_{y y} \\
{[\mathrm{MPa}]}\end{array}$ & $\rho_{\mathrm{iso}} / \rho_{0}$ & $\rho_{\mathrm{iso}} \kappa_{x x}$ & $\begin{array}{c}\lambda_{f} R_{\mathrm{sh}} \\
\text { FEM }\end{array}$ & $\begin{array}{c}\lambda_{f} R_{\mathrm{sh}} \\
\text { Difference FEM vs. theory }\end{array}$ & $\begin{array}{c}\lambda_{p} R_{\mathrm{sh}} \\
\text { FEM }\end{array}$ & $\begin{array}{c}\lambda_{p} R_{\mathrm{sh}} \\
\text { Difference FEM vs. theory }\end{array}$ \\
\hline 17 & 55 & 0 & 0 & 1.000000 & 1.00000 & 1.264527 & $-0.04 \%$ & 0.796063 & $-0.02 \%$ \\
17 & 55 & -5 & 5 & 0.999976 & 1.006929 & 1.262001 & $-0.04 \%$ & 0.800431 & $-0.02 \%$ \\
17 & 55 & -50 & 50 & 0.997613 & 1.071608 & 1.236262 & $-0.04 \%$ & 0.838836 & $-0.02 \%$ \\
17 & 55 & -500 & 500 & 0.723332 & 2.337100 & 0.641383 & $-0.03 \%$ & 1.068508 & $-0.03 \%$ \\
17 & 55 & 500 & -500 & 0.723332 & 0.427881 & 1.180510 & $-0.06 \%$ & 0.280305 & $-0.02 \%$ \\
17 & 55 & -500 & -400 & 0.972803 & 1.073496 & 1.204879 & $-0.04 \%$ & 0.819107 & $-0.02 \%$ \\
17 & 55 & 400 & 500 & 1.022421 & 1.069814 & 1.267640 & $-0.04 \%$ & 0.858564 & $-0.02 \%$ \\
17 & 55 & -400 & -500 & 0.972803 & 0.931536 & 1.254778 & $-0.04 \%$ & 0.731615 & $-0.02 \%$ \\
17 & 55 & 500 & 400 & 1.022421 & 0.934742 & 1.317534 & $-0.04 \%$ & 0.771065 & $-0.02 \%$ \\
55 & 17 & -500 & 500 & 0.723332 & 2.337100 & 0.280305 & $-0.02 \%$ & 1.180511 & $-0.06 \%$ \\
\hline
\end{tabular}

geometries in the wide range of mechanical stress up to $\pm 640 \mathrm{MPa}$. In an experiment the silicon wafer would very likely break at smaller stress levels, and the linear piezoresistive theory would describe the measured results insufficiently. Nevertheless, we chose the large stress to show the excellent agreement between analytical theory and numerical simulation.

The mechanical stress sensitivity at small stress is plotted in Figure 13, where we note a symmetry when $\theta_{f}$ and $\theta_{p}$ are swapped. Offset-free devices have $\theta_{f}=\theta_{p}$. They correspond to the black line and this line goes right through the global maximum of the function at the point $\theta_{f}=\theta_{p}=22.5^{\circ}$. This is one distinct difference to rectangular devices from Figure 5. This maximum sensitivity is $48.3501 \% / \mathrm{GPa}$, which is more than $3 \%$ larger than the maximum sensitivity with systematic offset in Figure 5 and nearly 6\% larger than for offsetfree rectangular devices in Figure 5. The mechanical stress sensitivity of offset-free circular devices is shown in Figure 14. The examples of rectangular versus circular devices show that different shapes of devices lead to different maximum achievable stress sensitivities. This is another distinct difference to Hall plates, where all shapes of devices, which can be derived from each other by conformal mapping, lead to the same magnetic sensitivity. However, there is another similarity between Hall plates and circular pseudo-Hall devices: at constant supply voltage their sensitivity to magnetic field and mechanical stress, respectively, is maximized, if the input and output resistances are identical to $\sqrt{2}$ squares: $\lambda_{f 0}=\lambda_{p 0}=$ $\sqrt{2}$ (compare Figure 14 with [12]). Yet this similarity does not hold for rectangular devices or inverted Greek crosses (see Figure 18(a)).

Figure 15 shows the relative change of resistances of the equivalent resistor circuit (ERC) for offset-free circular devices from Figure 12 with $\lambda_{f 0}=\lambda_{p 0}$, when $\sigma_{x x}-\sigma_{y y}=$ $-1 \mathrm{MPa}$ is applied and $\sigma_{x x}+\sigma_{y y}=\sigma_{x y}=0$. Low $\mathrm{p}$-doped silicon is assumed with $\pi_{44}=1.381 / \mathrm{GPa}$. Obviously $\bar{R}_{D}$ does not change while $\bar{R}_{H f}$ and $\bar{R}_{H p}$ change with opposite signs and equal magnitude. Their maximum stress sensitivity is $\pm 69.6384 \% / \mathrm{GPa}$ and it occurs at $\theta_{f}=\theta_{p}=27.3364^{\circ}$. For large contacts with $\theta_{f}=\theta_{p}=5^{\circ}$ the resistances $\bar{R}_{H f}$ and $\bar{R}_{H p}$ change less, only $\pm 43.5873 \% / \mathrm{GPa}$. Both cases were checked and verified by finite element simulations. The stress sensitivities of long resistor stripes of the same doping are $\partial R_{[110]} / \partial\left(\sigma_{x x}-\sigma_{y y}\right)=-\partial R_{[\overline{1} 10]} / \partial\left(\sigma_{x x}-\sigma_{y y}\right)=R_{0} \pi_{44} / 2=$ $69.05 \% / \mathrm{GPa} \times R_{0}$ (for stripes with current flowing in $x, y$ directions $)$ and $\partial R_{[100]} / \partial\left(\sigma_{x x}-\sigma_{y y}\right)=\partial R_{[010]} / \partial\left(\sigma_{x x}-\sigma_{y y}\right)=0$ (for stripes with current flowing in $\langle 100\rangle$-directions). Hence, the diagonal resistors $\bar{R}_{D}$ in the ERC behave like resistor stripes in diagonal direction $\langle 100\rangle$ : they do not change versus stress. However, the "horizontal" resistors $\bar{R}_{H p}$ in the ERC have a stress drift that depends on the size of the contacts. For medium sized contacts their stress drift can even slightly exceed the stress drift of resistor stripes in [110] direction, but for small or large contacts their stress drift is significantly lower. Therefore, in general the stress dependence of the resistors in the ERC between any two contacts is different from the stress dependence of simple resistor stripes aligned in the same direction between said contacts. Yet, for circular devices with medium sized contacts the difference is moderate.

4.4. Offset-Free Circular Devices with Point-Sized Contacts at Small Stress. From Figure 12(a) we see that this limit means $\pi / 2-\theta_{f}-\theta_{p} \rightarrow 2 d \theta$ with small $d \theta$. However, we discuss only the offset-free case $\theta_{f}=\theta_{p} \rightarrow \pi / 4-d \theta$. For small stress $\rho_{\text {iso }} \kappa_{x x} \rightarrow 1-\left(\pi_{44} / 2\right)\left(\sigma_{x x}-\sigma_{y y}\right)$. Inserting this into (26b) gives the differential

$$
\kappa \longrightarrow d \kappa=2\left(-4 \pi_{44}\left(\sigma_{x x}-\sigma_{y y}\right)\right)^{1 / 4},
$$




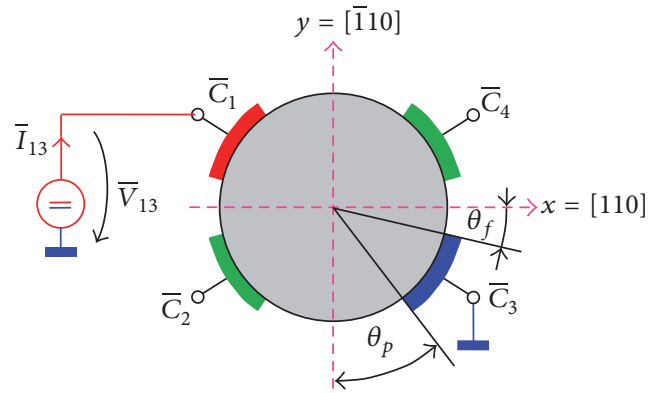

(a) Circular pseudo-Hall device with two perpendicular mirror-symmetry axes $x$ and $y$. It can be used for the measurement of $\sigma_{x x}-\sigma_{y y}$ on a chip made of (100)-silicon. The device is supplied with electrical power at the contacts $\bar{C}_{1}-\bar{C}_{3}$. The output signal is tapped between $\bar{C}_{2}$ and $\bar{C}_{4}$. The equivalent resistor circuit (ERC) is identical to Figure 6
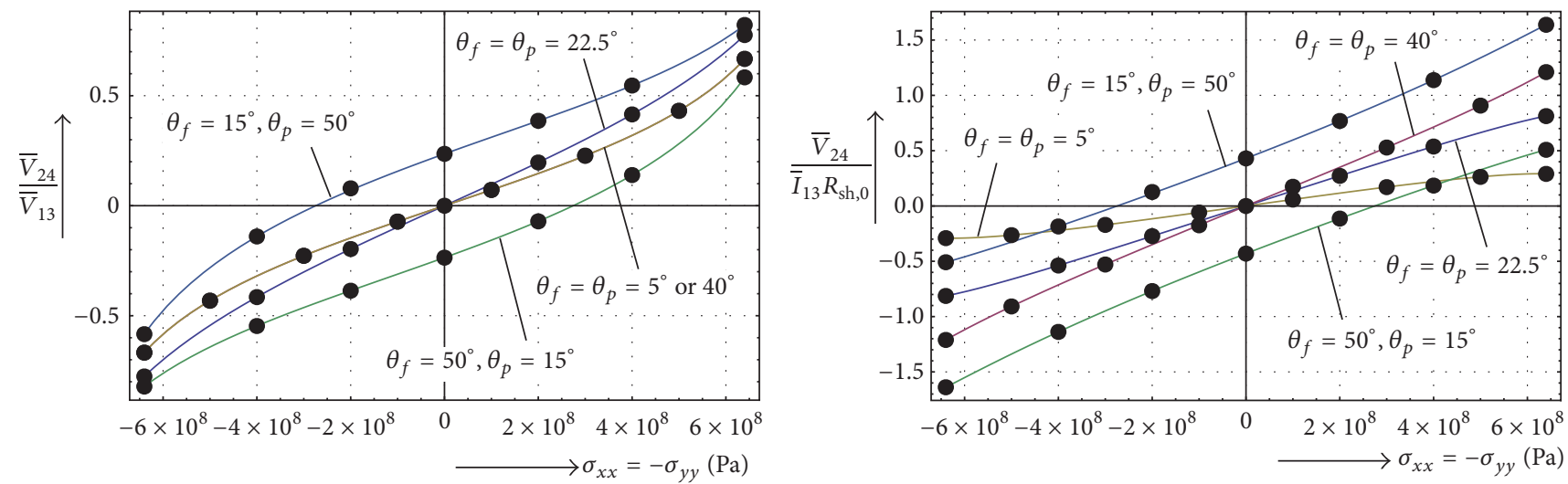

(b) Output voltage of the circular device from (a) versus biaxial inplane mechanical stress $\sigma_{x x}=-\sigma_{y y}$ with $\sigma_{x y}=0$. The left plot shows the ratio of output over supply voltage. The right plot shows the ratio of transimpedance over sheet resistance at zero stress $R_{\text {sh, }, 0}$. The solid curves are computed with the analytical theory. The black dots denote results form 29 finite element simulations with $\pi_{11}=6.6 \times 10^{-11} / \mathrm{Pa}, \pi_{12}=-1.1 \times 10^{-11} / \mathrm{Pa}$, and $\pi_{44}=138.1 \times 10^{-11} / \mathrm{Pa}($ as in low $\mathrm{p}$-doped silicon $)$

FIGURE 12

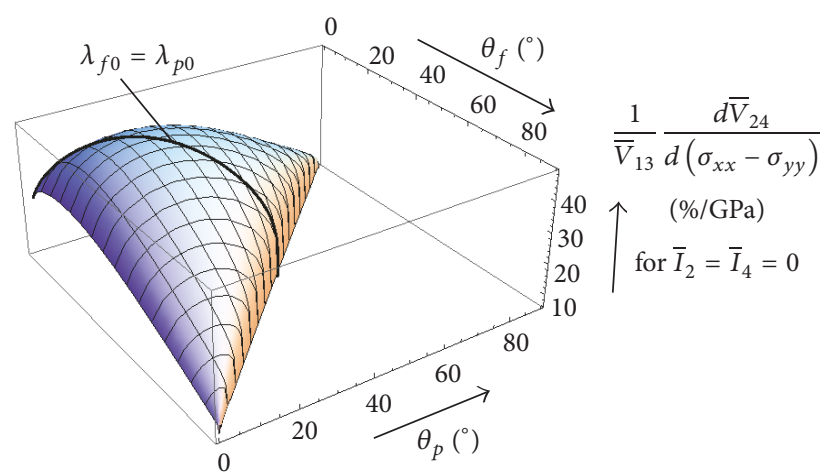

(a)

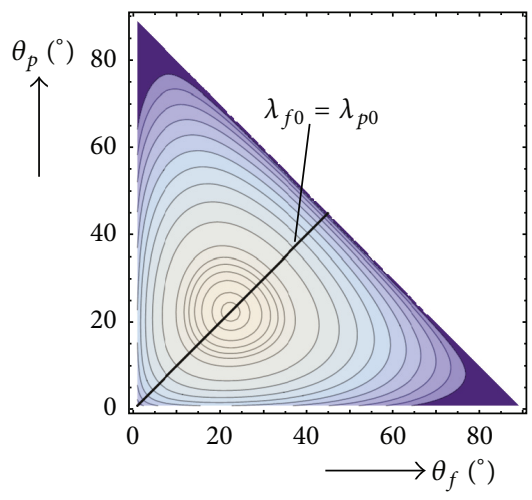

(b)

FIGURE 13: Sensitivity of output voltage $\bar{V}_{24}$ versus mechanical stress $\sigma_{x x}-\sigma_{y y}$ (normalized to supply voltage $\bar{V}_{13}$ ) for a circular device of Figure 12. All other components of the stress tensor vanish, as well as $\sigma_{x x}+\sigma_{y y}=0$. The plots are graphical representations of (26b), (28b), (28c), (18a)-(18i), and (19b) in the limit of small stress and with the piezoresistive coefficient $\pi_{44}=1.381 /$ GPa for low $\mathrm{p}$-doped silicon. Largest stress sensitivities are obtained for contact size $\theta_{f}=\theta_{p}=22.5^{\circ}$ with a flat maximum. The black curve denotes offset-free devices with $\lambda_{f 0}=\lambda_{p 0}$ at zero stress; they are the only ones, where $\bar{V}_{24}=0$ at zero stress. The black curve goes through the maximum of the surface. The contours in (b) are for $48.25,48,47.5,47,46.5,46,45,42.5,40, \ldots, 22.5,20 \% / \mathrm{GPa}$. In (a) the steep slopes of the surface near $\theta_{f}<1^{\circ}$ and $\theta_{p}<1^{\circ}$ are only sketched due to numerical problems. 


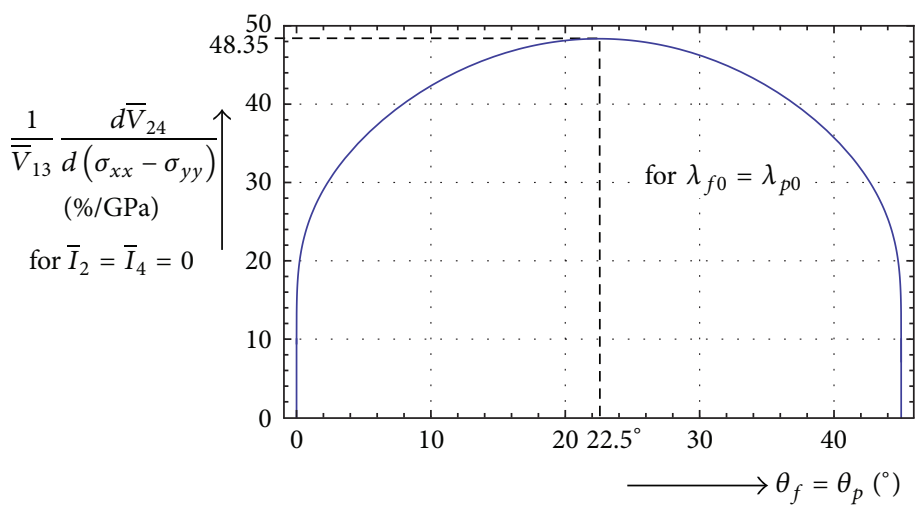

FIGURE 14: Sensitivity of output voltage $\bar{V}_{24}$ versus mechanical stress $\sigma_{x x}-\sigma_{y y}$ (normalized to supply voltage $\bar{V}_{13}$ ) for offset-free circular devices of Figure 13. All other components of the stress tensor vanish, as well as $\sigma_{x x}+\sigma_{y y}=0$. The plot is a graphical representation of (26b), (28b), (28c), (18a)-(18i), and (19b) in the limit of small stress and with the piezoresistive coefficient $\pi_{44}=1.381 /$ GPa for low p-doped silicon. Largest stress sensitivity of $48.3501 \% / \mathrm{GPa}$ is obtained for aspect ratios $\theta_{f}=\theta_{p}=22.5^{\circ}$ which means $\lambda_{f 0}=\lambda_{p 0}=\sqrt{2}$ and this is also the number of squares between supplies or sense contacts. The curve is mirror symmetric to $\theta_{f}=\theta_{p}=22.5^{\circ}$.

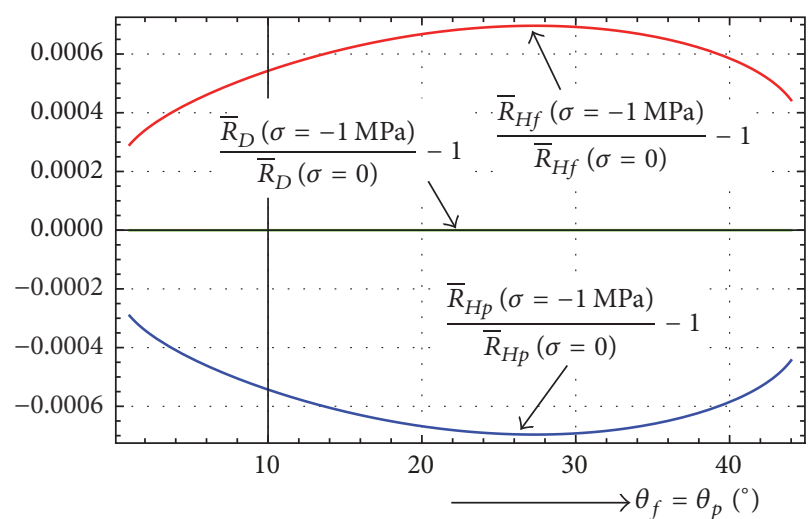

FIGURE 15: Relative change of resistances of the equivalent resistor circuit (ERC) for offset-free $\left(\lambda_{f 0}=\lambda_{p 0}\right)$ symmetric circular devices from Figure 12. $\sigma=\sigma_{x x}-\sigma_{y y}=-1 \mathrm{MPa}$ is applied and $\sigma_{x x}+\sigma_{y y}=\sigma_{x y}=0$. For $\pi_{44}=1.381 / \mathrm{GPa}$.

whereby we used $L(x) \rightarrow 1 / \cosh ^{2}(\pi x / 2-\ln 2)$ for large $x$ (see $[10])$ and we made a series in the small stress terms. With (28b) and (28c) we get

$$
\begin{aligned}
& k \longrightarrow \\
& 3- 2 \sqrt{2}+(3 \sqrt{2}-4)\left(2 d \theta-\frac{d \kappa^{4}}{64}\right) \\
&+\frac{(-16+11 \sqrt{2}) d \theta d \kappa^{4}}{64} \\
& \frac{s}{\ell}=\frac{s_{0}}{\ell_{0}} \longrightarrow \\
& \frac{F\left(1-2 \sqrt{2} d \theta-\sqrt{2} d \theta\left(\kappa^{4} / 64\right), k\right)}{K(k)}
\end{aligned}
$$

We plug this into (18i) and get

$$
w_{3} \longrightarrow 1-8 \sqrt{2} d \theta-\frac{\sqrt{2} d \theta \kappa^{4}}{16}
$$

Plugging (29b) and (30a) into (18d)-(18h) gives

$$
\left.\begin{array}{l}
\lambda_{f} \\
\lambda_{p}
\end{array}\right\} \rightarrow \frac{\pi}{\ln (4 / d \theta)}\left(1+\left\{\begin{array}{l}
+ \\
-
\end{array}\right\} \frac{\pi_{44}\left(\sigma_{x x}-\sigma_{y y}\right)}{\ln (4 / d \theta)}\right)
$$

In the limit $d \theta \rightarrow 0$ we get the transimpedance with (19c)

$$
\begin{aligned}
\frac{1}{I_{13}} \frac{d V_{24}}{d\left(\sigma_{x x}-\sigma_{y y}\right)} & \longrightarrow \\
\frac{2}{\pi} \pi_{44} R_{\mathrm{sh}} & \cong 0.63662 \times \pi_{44} R_{\mathrm{sh}}
\end{aligned}
$$

Equation (31) was checked by an FEM simulation. Comparing (31) with (25a) we note that in the limit of point-sized contacts the current related mechanical stress sensitivity of the disk shaped device is $8.6 \%$ smaller than for the square shaped device. Conversely, the current related magnetic sensitivity of both devices is identical, namely, $\mu_{H} R_{\text {sh }}$, according to (3) $\left(G_{H}=1\right.$ for point-sized contacts). 


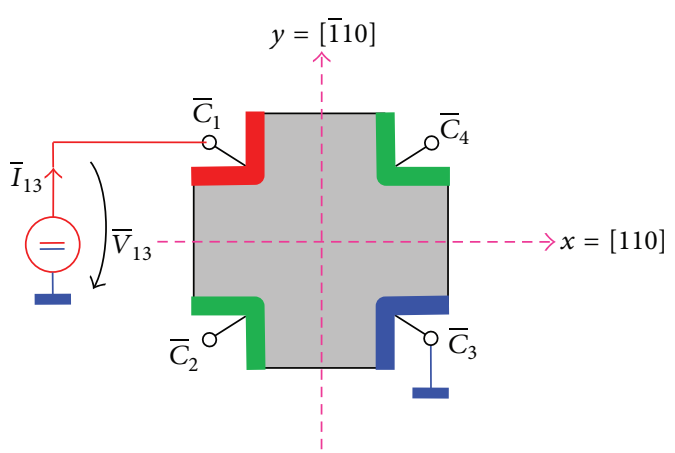

Figure 16: Pseudo-Hall device with two perpendicular mirrorsymmetry axes $x$ and $y$ : inverted Greek cross geometry. It can be used for the measurement of $\sigma_{x x}-\sigma_{y y}$ on a chip made of (100)-silicon. The device is supplied with electrical power at the contacts $\bar{C}_{1}-\bar{C}_{3}$. The output signal is tapped between $\bar{C}_{2}$ and $\bar{C}_{4}$. The equivalent resistor circuit (ERC) is identical to Figure 6.

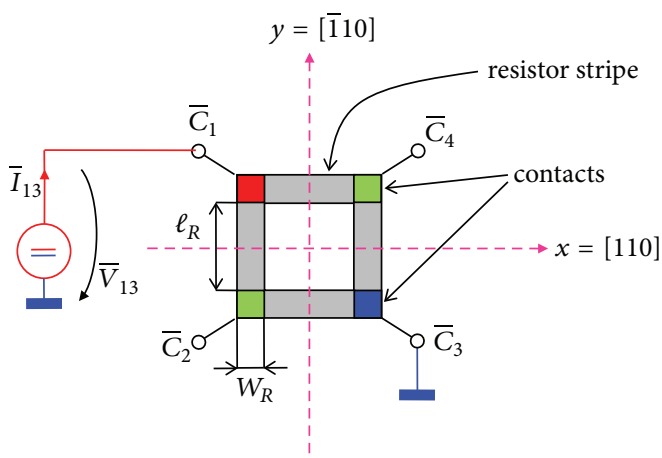

FIgURE 17: Wheatstone bridge with two perpendicular mirrorsymmetry axes $x$ and $y$ comprises four resistor stripes. It can be used for the measurement of $\sigma_{x x}-\sigma_{y y}$ on a chip made of (100)silicon. The device is supplied with electrical power at the contacts $\bar{C}_{1}-\bar{C}_{3}$. The output signal is tapped between $\bar{C}_{2}$ and $\bar{C}_{4}$. Note the similarity of the Wheatstone bridge with the inverted Greek cross of Figure 16 when the inner square of the bridge arrangement is filled with resistive material.

4.5. Inverted Greek Cross with Four Large Contacts. The device geometry is shown in Figure 16. In the piezoresistive case the device is effectively stretched in $x$-direction, which gives two perpendicular mirror symmetries. A numerical investigation with finite element simulation was done. Its results are shown in comparison with the other devices discussed so far in Figure 18.

4.6. Optimization of Stress Sensor Devices. Let us compare the stress sensitivity (25a) with a conventional Wheatstone bridge circuit with four simple stripes of resistors oriented in $x$ - and $y$-directions (see Figure 17). The stress dependence of these resistors is given by (6a) and (6b) if we assume that the stripes are much longer than wide. Thus, at constant input current the bridge output voltage at small stress is

$$
V_{\text {out }}=I_{\text {in }}\left(\sigma_{x x}-\sigma_{y y}\right) \pi_{44} R_{\mathrm{sh}} \frac{1}{2} \frac{\ell_{R}}{W_{R}},
$$

where $\ell_{R} / W_{R}$ is the number of squares of each resistor (i.e., the aspect ratio of each resistance stripe). A comparison of (25a) and (32) shows that the output voltage of the Wheatstone bridge is larger if each resistor has at least 1.4 squares. However, the ratio $V_{\text {out }} / I_{\text {in }}$ is not an appropriate measure to select optimum sensors, because the pseudoHall device of (25a) has infinite input and output resistance whereas the Wheatstone bridge has finite resistance. The resistance of the pseudo-Hall device diverges logarithmically when the contact size goes to zero (see (22a), (30b)). Hence, if we want to force a current through this sensor we would need infinite supply voltage and the thermal noise at the output would also increase unboundedly. Conversely, if the supply voltage is limited, the sensor output voltage goes logarithmically towards zero (see (22b)). In practice a circuit designer chooses the impedance level of the sensor circuit first to trade off current consumption and noise, and under this constraint he looks for the sensor geometry that maximizes the signalto-noise ratio (this is explained in [12]). For the Wheatstone bridge sensor this means that $R_{\mathrm{in}}=R_{\mathrm{sh}} \ell_{R} / W_{R}$ is fixed and then $V_{\text {out }}$ is maximized. If the sensor is operated from a battery or a regulated on-chip supply voltage, then the input voltage is also limited, and there is no degree of freedom left for optimization: $V_{\text {out }}=V_{\text {in }}\left(\sigma_{x x}-\sigma_{y y}\right) \pi_{44} / 2$. Conversely, if the sensor is powered by an off-chip supply with "unlimited" power, the relevant limit is maximum power dissipation $P_{d}$ and thermal destruction inside the sensor while $R_{\text {in }}$ can be chosen arbitrarily. Then we get $V_{\text {out }}=\sqrt{P_{d} R_{\text {in }}}\left(\sigma_{x x}-\sigma_{y y}\right) \pi_{44} / 2$ for the Wheatstone bridge and the output thermal noise voltage is proportional to $\sqrt{R_{\text {in }}}$.

For a device with finite contacts from Figure 12(a) we can set the impedance by choosing an appropriate thickness $t_{d}$ (see (19a)). Alternatively we could connect two (or several) devices in parallel to effectively double (or multiply) their thickness. The resistivity should be low, because this means low doping and this maximizes the piezoresistive coefficients [50]. Yet, in silicon the doping should also not be lower than $10^{15} / \mathrm{cm}^{3}$, because then mobile ions in the interfaces between various layers on the chip may give rise to lifetime drift [51]. For battery operation the signal should be maximized at fixed supply voltage and impedance. This leads to the maximum of Figure 14, with $V_{\text {out }}=V_{\text {in }}\left(\sigma_{x x}-\sigma_{y y}\right) \pi_{44} \times 0.350109$. Thus, at equal supply voltage and supply current the output voltage of an offset-free pseudo-Hall device from Figure 12(a) is 30\% smaller than the output voltage of a Wheatstone bridge sensor $(0.35 / 0.5=0.70=1-0.30)$. If the offset-free pseudoHall device does not have optimum contact size as in the maximum in Figure 14, its output voltage is even lower. Thus, stress sensitivity is not a strong argument for pseudo-Hall devices. Their advantage over Wheatstone bridges is their smaller size.

Figures 18(a) and 18(b) showthe output voltage over input voltage and input current, respectively, for several device geometries with finite contacts: rectangular devices from Figure 6, circular devices fromFigure 12, and the inverted Greek cross from Figure 16. Only offset-free devices are considered. The piezoresistive coefficient $\pi_{44}=1.381 / \mathrm{GPa}$ for low p-doped silicon was used. We assume low stress $\sigma_{x x}=-\sigma_{y y}$ and vanishing stress $\sigma_{x y}$. Then the output signal depends only on $\sigma_{x x}-\sigma_{y y}$. In Figure 18(a) we see 


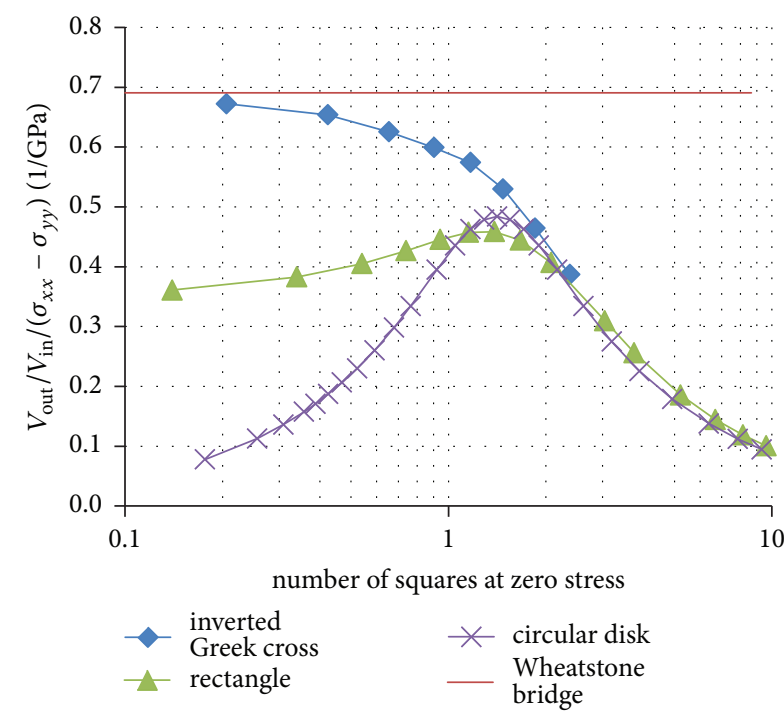

(a)

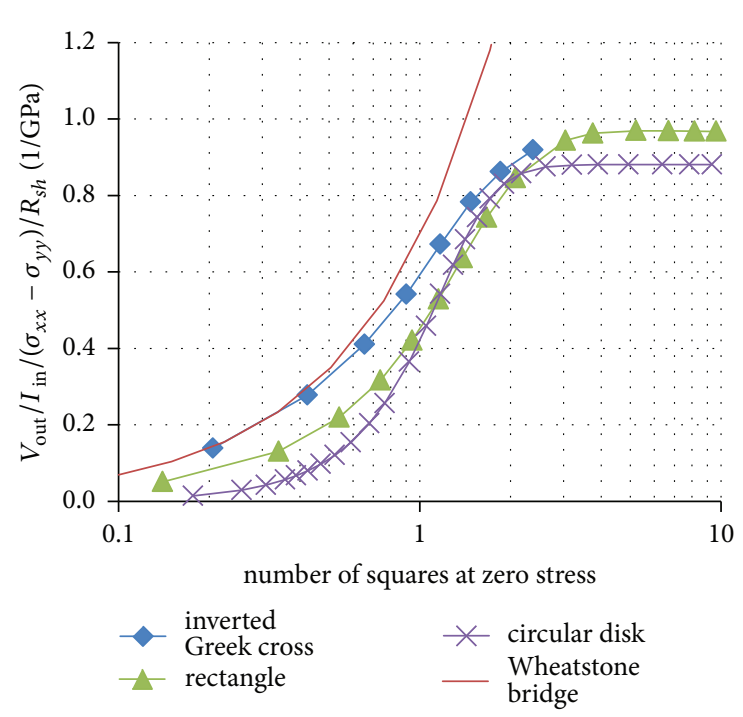

(b)

FIGURE 18: Comparison of (a) supply voltage related mechanical stress sensitivity, and (b) supply current related stress sensitivity of pseudoHall stress sensors with different shapes. Piezoresistive coefficient $\pi_{44}=1.381 / \mathrm{GPa}$ for low $\mathrm{p}$-doped silicon was assumed. Only offset-free devices with $\lambda_{f 0}=\lambda_{p 0}$ were considered. For the pseudo-Hall devices the number of squares is $R_{\mathrm{in}} / R_{\mathrm{sh}}=1 / \lambda_{f}+1 / \lambda_{p}$ and for the Wheatstone bridge it is $R_{\mathrm{in}} / R_{\mathrm{sh}}=\ell_{R} / W_{R}$.

that the Wheatstone bridge has the largest voltage related stress sensitivity, independent of the number of squares of its resistors. In the limit of low number of squares (i.e., low input resistance) the inverted Greek cross has the same stress sensitivity as the Wheatstone bridge. This agrees with common sense, because in this limit of the inverted Greek cross the supply and sense contacts face each other with small spacing, so that the current flows in pure $x$ - and $y$ directions like in a Wheatstone bridge circuit. The rectangular and the circular devices both show similar maxima at similar internal resistance. It is interesting to see that rectangular and circular piezoresistive devices behave similar, yet the inverted Greek cross is quite different, whereas for Hall plates their magnetic sensitivities are all identical when the abscissa axis gives the number of squares. In Figure 18(b) we see the current related stress sensitivity, which rises monotonously with the number of squares for all devices. However, the maximum of circular disks is smaller than the maxima of rectangle and inverted Greek cross (which are of course identical because in this limit their shapes are also identical). The smaller stress sensitivity of pseudo-Hall devices seems to have two reasons: (i) the diagonal resistor $2 \bar{R}_{D}$ in the equivalent resistor circuit loads the output and (ii) depending on contact size the stress sensitivity of the other resistors $\bar{R}_{H f}$ and $\bar{R}_{H p}$ in the equivalent resistor circuit is smaller than for resistor stripes in pure $x$ - or $y$-directions (see Figure 15).

At the end of this section, we mention how the devices of Figures 6, 12, and 16 can be used to measure $\sigma_{x y}$, if it holds $\sigma_{x x}-\sigma_{y y}=0$. In a reference frame $\Sigma^{\prime}\left(x^{\prime}, y^{\prime}\right)$, which is rotated against $\Sigma(x, y)$ by $-45^{\circ}$ the stress tensor reads $\sigma_{x^{\prime} x^{\prime}}=$ $\left(\sigma_{x x}+\sigma_{y y}\right) / 2-\sigma_{x y}, \sigma_{y^{\prime} y^{\prime}}=\left(\sigma_{x x}+\sigma_{y y}\right) / 2+\sigma_{x y}$, and $\sigma_{x^{\prime} y^{\prime}}=$

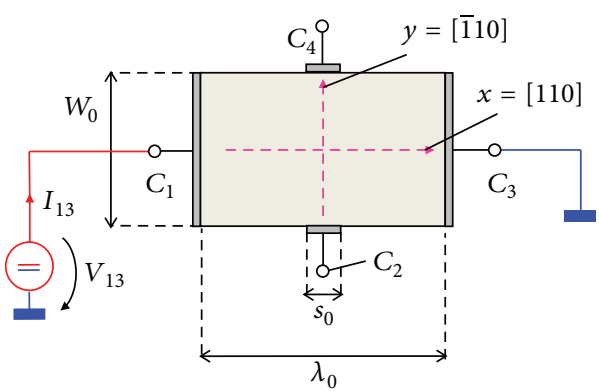

FIGURE 19: Rectangular Hall plate in (100)-silicon with two perpendicular mirror-symmetry axes $x=[110]$ and $y=[\overline{1} 10]$. An input voltage is forced between contacts $C_{1}$ and $C_{3}$ and an output voltage is tapped between $C_{2}-C_{4}$.

$\left(\sigma_{x x}-\sigma_{y y}\right) / 2$. Inserting this into the resistivity tensor of cubic crystals shows that we only need to rotate the devices by $-45^{\circ}$ and replace $\left(\sigma_{x x}-\sigma_{y y}\right) \pi_{44} / 2$ by $\sigma_{x y}\left(\pi_{12}-\pi_{11}\right)$ in (17a) and (17b).

If both $\sigma_{x y} \neq 0$ and $\sigma_{x x}-\sigma_{y y} \neq 0$, then the proposed theory cannot describe the ERC and the output signals.

\section{Devices with Mirror Symmetries to $x$ - and $y$-Axes and Contacts Lying on the $x$ - and $y$-Axes, at Zero Shear Stress $\sigma_{x y}=0$}

An example of such a device with rectangular geometry is shown in Figure 19. It is complementary to the device in Figure 6, because contacts and isolating boundary are swapped. Also a different equivalent resistor circuit applies 
TABLE 3: Comparison of $G_{H 0}$ between this work and [47]. The reference data of [47] were obtained by the FEM simulation from Figure 20: it is the slopes of the curves in the origin inserted into (33) and solved for $G_{H 0}$. In the FEM simulation the parameters of the Hall plate from Figure 19 were $\ell_{0} / W_{0}=2$ and $s_{0} / \ell_{0}=10^{-7}$. The difference between both is small and it seems to come from the nonlinearity of the curves (compare first and last lines).

\begin{tabular}{lccccccc}
\hline $\begin{array}{l}\sigma_{x x}=-\sigma_{y y} \\
{[\mathrm{MPa}]}\end{array}$ & $\rho_{x x} / \rho_{y y}$ & $\rho_{\text {iso }} \kappa_{x x}$ & $C_{H} B_{z} / \rho_{y y}$ & $V_{42} /\left(V_{13} C_{H} B_{z} / \rho_{y y}\right)$ & $\begin{array}{c}G_{H 0} \\
([47])\end{array}$ & $\begin{array}{c}G_{H 0} \\
\text { (this work) }\end{array}$ & $\begin{array}{l}\text { difference } \\
4411.76\end{array}$ \\
0.25 & 2 & 0.01 & 1.3504349 & 0.67521745 & 0.67531489 & $-144 \mathrm{ppm}$ \\
0 & 1 & 1 & 0.01 & 0.4650187 & 0.93003740 & 0.93006014 & $-24.5 \mathrm{ppm}$ \\
-4411.76 & 4 & 0.5 & 0.01 & 0.1246212 & 0.99696960 & 0.99697235 & $-2.76 \mathrm{ppm}$ \\
4411.76 & 0.25 & 2 & 0.0001 & 1.3506300 & 0.67531500 & 0.67531489 & $0.159 \mathrm{ppm}$ \\
\hline
\end{tabular}

to the new device (see [10]). Nevertheless, the quantities $\lambda_{f}$, $\lambda_{p}$ are identical to (18d) and(18e) (see [10]). At zero magnetic field the voltage between contacts $C_{2}-C_{4}$ does not depend on $\sigma_{x x}$ and $\sigma_{y y}$ due to symmetry. However, $\lambda_{f}$ and $\lambda_{p}$ depend on $\sigma_{x x}$ and $\sigma_{y y}$. And according to (4) the Hall-geometry factor $G_{H 0}$ for small magnetic field also depends on $\sigma_{x x}$ and $\sigma_{y y}$. Hence, the magnetic sensitivity of the Hall plate changes with mechanical stress due to the piezoresistance effect! Note that this is a different effect than the well-known piezo-Hall effect [52], which describes the change of a material property-the Hall coefficient $C_{H}$-caused by mechanical stress. In our case the piezoresistivity leads to a small net change in Hall-plate geometry described by the affine transformation (12a)-(12c) and this slightly changes the Hall-geometry factor. Therefore Hall plates of identical material but different geometry also have slightly different drift of magnetic sensitivity versus mechanical stress.

We verify this model by comparison with Figure 3 of [47]. There the authors plotted the Hall output voltage versus magnetic field for several cases of highly anisotropic resistivity for a Hall plate with $\ell_{0} / W_{0}=2$ and $s_{0} / \ell_{0} \rightarrow 0$, i.e., point-sized output contacts. For a meaningful comparison we need accurate numerical data, which were not given in [47]. Therefore we repeated three calculations of [47] with a finite element simulation (FEM) with COMSOL MULTIPHYSICS having 1.4 million elements and Lagrange multipliers. The results are shown in Figure 20: with the naked eye these curves match perfectly with the respective curves in Figure 3 of [47]. In the FEM we used the resistivity tensor of (5) with (6a)-(6c) and with the piezoresistive coefficients for low ndoped silicon $\left(\pi_{11}=-1.022 / \mathrm{GPa}, \pi_{12}=0.534 / \mathrm{GPa}\right.$, and $\left.\pi_{44}=-0.136 / \mathrm{GPa}\right)$. For a stress $\sigma_{x x}=-\sigma_{y y}= \pm 4.41176 \mathrm{GPa}$ this gives very strong anisotropy $4 \rho_{x x}=\rho_{y y}$ and $\rho_{x x}=4 \rho_{y y}$, respectively. In the minor diagonal of the resistivity tensor we added the antireciprocal Hall-terms $\pm C_{H} B_{z}$. Next we used the FEM to get exact numerical data of the slopes of the curves at small magnetic field. According to theory these slopes are

$$
\frac{V_{42} / V_{13}}{C_{H} B_{z} / \rho_{y y}}=\frac{W_{0}}{\ell_{0}} \frac{\rho_{y y}}{\rho_{x x}} G_{H 0}
$$

from which one can obtain $G_{H 0}$ for the three cases. Table 3 compares these values from the FEM with $G_{H 0}$ according to the analytical theory (18d), (18e), and (18g)-(18l) inserted into

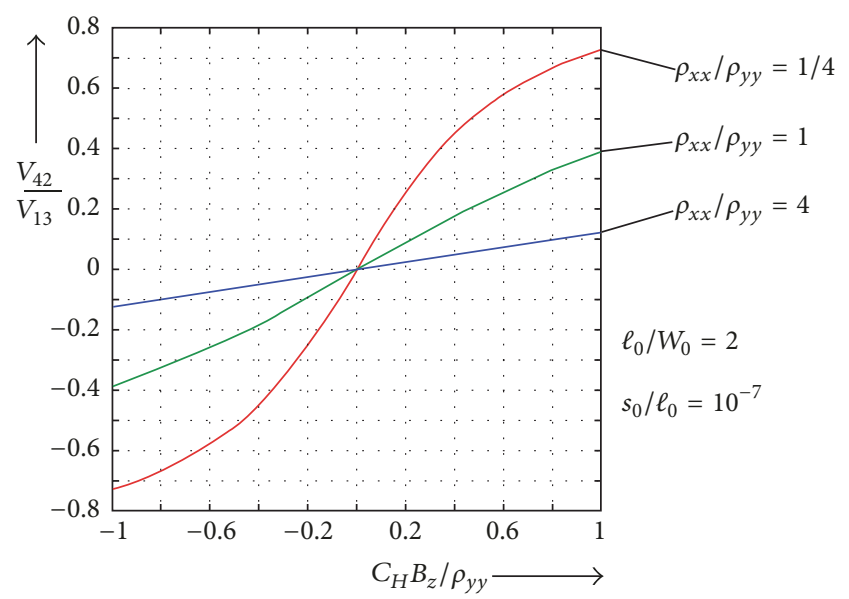

FIGURE 20: Output voltage over supply voltage of a Hall plate from Figure 19 with two squares input resistance and point-sized output contacts as obtained by a finite element simulation with COMSOL MULTIPHYSICS. The strong anisotropy of the conductivity tensor is obtained by large stress $\sigma_{x x}=-\sigma_{y y}= \pm 4.41176 \mathrm{GPa}$ in low ndoped silicon. The curves match with respective curves 1 (for $\gamma=0$ ) in Figure 3 of [47]. The slopes of the curves in the weak field limit agree with the analytical theory of the present work (see Table 3).

(4). Despite the large stress the agreement is up to $10^{-6}$ for small magnetic field.

Next we compute the piezoresistive drift of current related magnetic sensitivity $\partial G_{H 0} / \partial\left(\sigma_{x x}-\sigma_{y y}\right) / G_{H 0}$ for circular Hall plates of Figure 10(a) versus all possible contact geometries $\theta_{f}$ and $\theta_{p}$ (see Figure 21). This effect vanishes for symmetric devices with $\theta_{f}=\theta_{p}$ (except for very large stress). This was also checked by a finite element simulation for the case $\theta_{f}=$ $\theta_{p}=22.5^{\circ}$

Therefore, they should be a perfect choice if one wants to measure the piezo-Hall coefficient. Note that $\partial G_{H 0} / \partial\left(\sigma_{x x}-\right.$ $\left.\sigma_{y y}\right)=0$ for any device with $90^{\circ}$-symmetry-not only for circular ones. The proof is short: if $G_{H 0}$ increases by $\sigma_{x x}-$ $\sigma_{y y}>0$, then it decreases by $\sigma_{x x}-\sigma_{y y}<0$. However, the latter case is obtained by swapping $\sigma_{x x}$ with $\sigma_{y y}$, and obviously this cannot change $G_{H 0}$ if the device is $90^{\circ}$ symmetric. Therefore $G_{H 0}$ does not change at all under the action of $\sigma_{x x}-\sigma_{y y}$.

Conversely, if one pair of contacts is point-sized and the other one is close to $62.5^{\circ}$, Figure 21 shows a maximum 


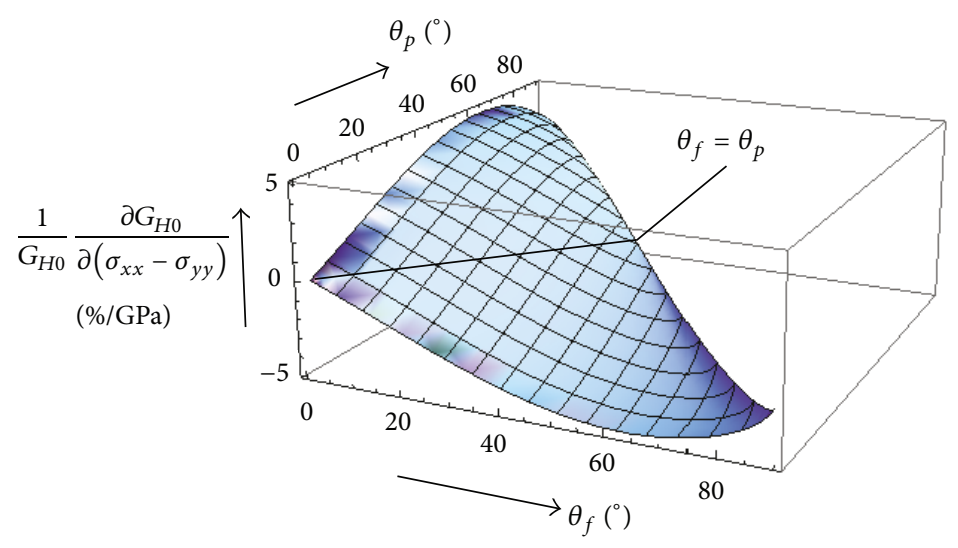

FigURE 21: $\partial G_{H 0} / \partial\left(\sigma_{x x}-\sigma_{y y}\right) / G_{H 0}$ for circular Hall plates of Figure $10(\mathrm{a})$ versus contact sizes $\theta_{f}$ and $\theta_{p}$. This is the piezoresistive influence on the current related magnetic sensitivity of the Hall plate. It vanishes for symmetric devices with $\theta_{f}=\theta_{p}$ and it is strongest for devices, where one contact pair is point-sized and the other contact pair has a half-aperture angle of $62.5^{\circ}$. The plot assumes low n-doped (100)-silicon with $\pi_{44}=-0.136 / \mathrm{GPa}$. The plot region $89.5^{\circ}<\theta_{f}+\theta_{p}<90^{\circ}$ is not shown due to numerical problems in its evaluation - for $\theta_{f}+\theta_{p}=90^{\circ}$; it holds $G_{H 0}=0$ (see Figure 13) and $\partial G_{H 0} / \partial\left(\sigma_{x x}-\sigma_{y y}\right) / G_{H 0}=0$.

piezoresistive magnetic sensitivity drift of $\partial G_{H 0} / \partial\left(\sigma_{x x}-\right.$ $\left.\sigma_{y y}\right) / G_{H 0}= \pm 4.961 \% / \mathrm{GPa}$. A numerical FEM simulation with stresses $0.1 \mathrm{MPa}, 1 \mathrm{MPa}, 10 \mathrm{MPa}, 100 \mathrm{MPa}$, and $1 \mathrm{GPa}$ was conducted for two cases: first current was sent through the large contacts while voltage was tapped at the point-sized contacts, and then the roles of the contacts were swapped: the agreement with the analytical theory was around $0.01 \%$. If such a device was used in a uniaxial stress state to characterize the piezo-Hall coefficient in low n-doped (100)silicon-which is $P_{12} \approx 40 \% / \mathrm{GPa}$-the $5 \% / \mathrm{GPa}$ from the piezoresistive effect would lead to a significant error in $P_{12}$, if one does not account explicitly for this effect. On the other hand, a comparison of magnetic sensitivities of two such Hall plates, one with large contacts along the $x$-axis and the other one with large contacts along the $y$-axis, could be used to determine the piezoresistive coefficient $\pi_{44}$ without measuring resistances. If one uses an isotropic biaxial stress state $\sigma_{x x}=\sigma_{y y}$ (e.g., in a wafer bow experiment [53]) to characterize the piezo-Hall effect, the current related magnetic sensitivity does not depend on the piezoresistance effect. In smart silicon Hall sensors with mechanical stress compensation the piezoresistance effect leads to a $\sigma_{x x}-\sigma_{y y}$ dependence of the magnetic sensitivity, whereas the piezoHall effect has a $\sigma_{x x}+\sigma_{y y}$ dependence: this leads to increased complexity of the compensation circuit as described in chapter 16.6.3 in [22].

In the original paper by Hälg [52] the macroscopic Hall plates had the geometry of Figure 19 with $\ell_{0}=10 \mathrm{~mm}$, $W_{0}=1.6 \mathrm{~mm}, s_{0}=0.1 \mathrm{~mm}, t_{d}=0.38 \mathrm{~mm}$ with low n-doping. At zero stress the Hall-geometry factor $G_{H 0}$ is 0.9601759 . He measured a piezo-Hall coefficient $P_{12}=$ $40 \cdots 45 \% / \mathrm{GPa}$ depending on the doping concentration. The change of $G_{H 0}$ due to piezoresistance in the (100)-plane is $0.275 \% / \mathrm{GPa}$, which gives a relative error of $0.7 \%$ in $P_{12}$. If we include pure strain effects after (16a) the relative error reduces by one tenth. This error is so small because a device with small geometry effects was chosen by Hälg $\left(G_{H 0} \approx 1\right)$.

\section{Comparison with Other Authors}

With (18a)-(18m) and the ERC we can describe the behavior of the device versus arbitrarily large inplane normal stress in any operating mode: in the Hall-operating mode of Figure 6 (with the supply contacts $\bar{C}_{1}-\bar{C}_{3}$ and the sense contacts $\bar{C}_{2}-\bar{C}_{4}$ ), or in the van der Pauw operating mode (with the supply contacts $\bar{C}_{1}-\bar{C}_{2}$ and the sense contacts $\bar{C}_{3}-\bar{C}_{4}$ as shown in Figure 5 in [10]), or in the grounded diagonal operating mode (with the positive supply contact $\bar{C}_{1}$ while $\bar{C}_{2}$ and $\bar{C}_{4}$ are grounded and the sense contact is $\bar{C}_{3}$, cf. [10]).

However, the body of (18a)-(18m) seems much more complicated than piezoresistive theories of other authors $[2,3,6,54]$. According to the principle of Occam's razor we should prefer the simplest way to explain phenomena in nature. Therefore, our theory only makes sense, if it has some advantage over simpler theories:

In Figure 2 it was already explained that the theory of [2] fails for devices with four extended contacts and low or moderate number of squares, whereas the theory proposed in this paper works for all rectangular and circular devices with two perpendicular mirror symmetries.

The theory of [54] uses an equivalent resistor circuit (ERC) that comprises only four resistors in a Wheatstone bridge configuration. Therefore it is not apt to describe van der Pauw measurements, where the device is supplied with energy at two neighboring contacts and the signal is tapped between the two other neighboring contacts. Instead, in [54] the authors focused on typical Hall-operating modes, where current flows between two nonneighboring contacts and signal is tapped between the other contacts. The stress dependence of the four resistors in the ERC is modeled identically to the stress dependence of individual resistor stripes, which are oriented at $45^{\circ}$ to the global current flow direction. No justification is given for this assumption in [54]. Nevertheless, it seems to have worked reasonably well, because the authors claim fair agreement with experiments (deviations up to $35 \%$ were reported in their Table 1). However, empirical 
investigations were reported only for square Hall plates with point-sized output contacts (although various crystal planes and current flow directions were examined). An extension to rectangular Hall devices is mentioned in two sentences, but not verified. In case of more general device geometries with extended contacts it is not self-evident, which directions we should assign to each resistor in the ERC: e.g., in a symmetric device like in Figure 2(b) one might guess that according to [54] the equivalent resistors of the ERC are aligned at $\pm 45^{\circ}$ to the vertical line; however, in a less symmetric device like in Figure 6 it is unclear if an equivalent resistor should be aligned at $\pm 45^{\circ}$ to the vertical line or differently (especially if $\ell_{0} \gg W_{0} \gg s_{0}$ ).

Up to the author's knowledge, no one has so far described the effect of piezoresistance on the magnetic sensitivity. The plots in Figure 3 of [47] implicitly contain this information, but the scale is too coarse to note these subtle effects there, and the authors have not mentioned this phenomenon in their text.

\section{Conclusion}

This paper displayed similarities and differences between the reciprocal piezoresistance effect and the antireciprocal Hall effect. Alleged analogies of former authors were found to be valid only for special shapes of devices and contacts. An analytical theory was developed for devices with two perpendicular mirror symmetries exposed to biaxial stress along the symmetry axes. The theory was applied to rectangular and circular devices. In both cases it was found that the output signal is strongest for specific sizes of contacts, and the maximum output signals at given stress differ for various shapes of devices. Recurring to a statement of Wick in [14] we may say that for Hall plates there is no ingenious shape to maximize their magnetic sensitivity but for van der Pauw stress sensors and pseudo-Hall devices there definitely exist shapes which have larger mechanical stress sensitivity than others. For example, circular devices offer $\sim 6 \%$ higher stress sensitivity than rectangular devices. An outstanding case is deep vertical Hall effect devices powered by voltage sources, where the potentials at floating contacts at zero magnetic field are not at all affected by mechanical stress in the cross-section plane.

It was also shown that the magnetic sensitivity of Hall plates with finite contacts has a small dependence on the piezoresistive effect, which adds to the piezo-Hall effect. The piezoresistance can be accounted for by an affine transformation, which slightly distorts the shape of the Hall plate. This leads to small changes in the number of squares of input and output resistances, which changes the Hall geometry factor and consequently also the magnetic sensitivity. This effect depends on the specific shape of the Hall plate. It vanishes for devices with $90^{\circ}$ symmetry in cubic crystals, and therefore these devices are well suited to characterize the piezo-Hall effect with high accuracy.

\section{Conflicts of Interest}

The author declares that there are no conflicts of interest.

\section{References}

[1] W. G. Pfann and R. N. Thurston, "Semiconducting stress transducers utilizing the transverse and shear piezoresistance effects," Journal of Applied Physics, vol. 32, no. 10, pp. 2008-2019, 1961.

[2] V. A. Gridchin, "Influence of the specimen geometry on the magnitude of the transverse piezo emf," Soviet Physics Journal, vol. 19, no. 4, pp. 457-460, 1976.

[3] A. V. Gridchin and V. A. Gridchin, "The four-terminal piezotransducer: theory and comparison with piezoresistive bridge," Sensors and Actuators A: Physical, vol. 58, no. 3, pp. 219-223, 1997.

[4] A. K. M. Mian, J. C. Suhling, R. C. Jaeger, and B. M. Wilamowski, "Evaluation of die stress using van der Pauw sensors," Applitcations of Experimental Mechanics to Electronic Packaging, vol. 226, pp. 59-67, 1997.

[5] P. J. A. Munter, "A low-offset spinning-current Hall plate," Sensors and Actuators A: Physical, vol. 22, no. 1-3, pp. 743-746, 1989.

[6] Y. Kanda and A. Yasukawa, "Hall-effect devices as strain and pressure sensors," Sensors and Actuators, vol. 2, no. C, pp. 283296, 1981.

[7] M. Doelle, D. Mager, P. Ruther, and O. Paul, "Geometry dependent sensitivity of planar plezoresistive stress sensors based on the pseudo-Hall effect," in Proceedings of the 18th IEEE International Conference on Micro Electro Mechanical Systems (MEMS '05), pp. 267-270, February 2005.

[8] L. J. van der Pauw, "Determination of resistivity tensor and Hall tensor of anisotropic conductors," Philips Research Reports, vol. 16, pp. 187-195, 1961.

[9] U. Ausserlechner, "Closed form expressions for sheet resistance and mobility from Van-der-Pauw measurement on $90^{\circ}$ symmetric devices with four arbitrary contacts," Solid-State Electronics, vol. 116, pp. 46-55, 2016.

[10] U. Ausserlechner, "Van-der-Pauw measurement on devices with four contacts and two orthogonal mirror symmetries," SolidState Electronics, vol. 133, pp. 53-63, 2017.

[11] U. Ausserlechner, "A method to compute the Hall-geometry factor at weak magnetic field in closed analytical form," Electrical Engineering, pp. 1-8, 2015.

[12] U. Ausserlechner, "The signal-to-noise ratio and a hidden symmetry of Hall plates," Solid-State Electronics, vol. 135, pp. 1423, 2017.

[13] H. T. Grahn, Introduction to Semiconductor Physics, chapter 6.3, World Scientific Publishing, 1999.

[14] R. F. Wick, "Solution of the field problem of the germanium gyrator," Journal of Applied Physics, vol. 25, no. 6, pp. 741-756, 1954.

[15] U. Ausserlechner, "Sensor arrangement having thermo-emf compensation," US patent US9829543, 2017.

[16] S. R. de Groot and P. Mazur, Nonequilibrium thermodynamics, chapter XIII, section 5, Dover Publications, New York, NY, USA, 1984.

[17] H. J. Carlin, "On the physical realizability of linear nonreciprocal networks," Proceedings of the IRE, vol. 43, no. 5, pp. 608-616, 1955.

[18] B. D. H. Tellegen, “The Gyrator, a new electric network element," Philips Research Reports, vol. 3, pp. 81-101, 1948.

[19] H. J. Carlin and A. B. Giordano, Network Theory: An Introduction to Reciprocal and Nonreciprocal Circuits, chapter 5, Prentice-Hall, New Jersey, NJ, USA, 1964. 
[20] J. M. Garg and H. J. Carlin, "Network theory of semiconductor hall-plate circuits," IEEE Transactions on Circuit Theory, vol. 12, no. 1, pp. 59-73, 1965.

[21] Y. Zou, J. Suhling, R. Jaeger, and H. Ali, “Three dimensional die surface stress measurements in delaminated and nondelaminated plastic packages," in Proceedings of the 48th Electronic Components and Technology Conference, pp. 1223-1234, Seattle, WA, USA, 1998.

[22] M. Motz and U. Ausserlechner, "Electrical compensation of mechanical stress drift in precision analog circuits," in Wideband Continuous-time $\Sigma \Delta$ ADCs, Automotive Electronics, and Power Management: Advances in Analog Circuit Design 2016, chapter 16, pp. 297-326, Springer International Publishing, 2016.

[23] D. A. Bittle, J. C. Suhling, R. E. Beaty, R. C. Jaeger, and R. W. Johnson, "Piezoresistive stress sensors for structural analysis of electronic packages," Journal of Electronic Packaging, Transactions of the ASME, vol. 113, no. 3, pp. 203-215, 1991.

[24] A. Mian, J. C. Suhling, and R. C. Jaeger, "The van der Pauw stress sensor," IEEE Sensors Journal, vol. 6, no. 2, pp. 340-356, 2006.

[25] J. F. Nye, Physical Properties of Crystals-Their Representation by Tensors and Matrices, vol. 36, chapter II, section 4, p. 44, Oxford Science Publications, Oxford, UK, 1985.

[26] W. Schnell, D. Gross, and W. Hauger, Technische Mechanik. Band 2: Elastostatik, chapter 2.2.2, Springer Berlin Heidelberg, 4th edition, 1992.

[27] G. Heppke and F. Schneider, "Elektrische Eigenschaften von rechteckigen Proben anisotroper Leiter," Annalen der Physik, vol. 29, no. 3, pp. 271-288, 1973.

[28] R. Terakado and T. Sakakibara, "Analysis of two-dimensional anisotropic regions by affine and conformal mappings," Proceedings of the IEEE, vol. 75, no. 6, pp. 844-846, 1987.

[29] H. Reiche, "Der Halleffect als Anisotropie des elektrischen Leiters," Wissenschaftliche Zeitschrift der Technischen Universität Dresden, vol. 12, no. 1, pp. 179-182, 1963.

[30] A. Sutor and R. Lerch, "New Model for a CMOS-Compatible Stress Sensor Based on the Anisotropy of the Piezoresistive Effect," in Proceedings of the 10th Intl Fair and Congress for Sensors, Transducers and Systems, pp. 29-34, May 2001.

[31] G. O. Coraucci, R. V. D. de Oliveira, V. Garcia, and F. Fruett, "A study of the geometrical correction factor and the membrane thickness on the sensitivity of the transversal piezoresistive pressure sensor," Journal of Integrated Circuits and Systems, vol. 5, no. 2, pp. 140-147, 2010.

[32] V. Gridchin, A. Gridchin, V. Lubimsky, and V. Besedin, "Experimental investigation of the Greek cross-shaped piezoresistive element," in Proceedings of the 1998 4th International Conference on Actual Problems of Electronic Instrument Engineering Proceedings (APEIE '98), vol. 1, pp. 113-118, Novosibirsk, Russia, September 1998.

[33] W. L. V. Price, "Electric potential and current distribution in a rectangular sample of anisotropic material with application to the measurement of the principal resistivities by an extension of van der Pauw's method," Solid-State Electronics, vol. 16, no. 7, pp. 753-762, 1973.

[34] K. A. Borup, K. F. F. Fischer, D. R. Brown, G. J. Snyder, and B. B. Iversen, "Measuring anisotropic resistivity of single crystals using the van der Pauw technique," Physical Review B: Condensed Matter and Materials Physics, vol. 92, no. 4, Article ID 045210, 2015.
[35] J. F. Nye, Physical Properties of Crystals. Their Representation by Tensors and Matrices, vol. 36, chapter VI, section 2, Oxford Science Publications, Oxford, UK, 1985.

[36] J. F. Nye, Physical Properties of Crystals. Their Representation by Tensors and Matrices, vol. 36, chapter VI, section 3.1, Oxford Science Publications, Oxford, UK, 1985.

[37] M. A. Hopcroft, W. D. Nix, and T. W. Kenny, "What is the Youngs Modulus of Silicon?" Journal of Microelectromechanical Systems, vol. 19, no. 2, pp. 229-238, 2010.

[38] W. Versnel, "Analysis of symmetrical Hall plates with finite contacts," Journal of Applied Physics, vol. 52, no. 7, pp. 46594666, 1981.

[39] R. S. Popovic, “The vertical Hall-effect device," IEEE Electron Device Letters, vol. 5, no. 9, pp. 357-358, 1984.

[40] U. Ausserlechner, "Hall Effect Devices with Three Terminals: Their Magnetic Sensitivity and Offset Cancellation Scheme," Journal of Sensors, vol. 2016, Article ID 5625607, 16 pages, 2016.

[41] R. Sunier, S. Taschini, O. Brand, T. Vancura, and H. Baltes, "Quasi-analytical study of offset voltage due to piezoresistive effect in vertical Hall devices by mapping techniques," in Proceedings of the IEEE International Solid-State Sensors and Actuators Conference, pp. 1582-1585, Boston, MA, USA, June 2003.

[42] K. Maenaka, "Piezoresistive effect in vertical Hall cell," IEEE Electronics Letters, vol. 29, no. 4, pp. 381-382, 1993.

[43] R. E. Newnham, Properties of Materials. Anisotropy, Symmetry, Structure, chapter 13.4, Oxford UP, Oxford, UK, 2005.

[44] J. M. Borwein and P. B. Borwein, Pi and the AGM-A Study in Analytical Number Theory and Computational Complexity, Canadian Mathematical Society Series of Monographs and Advanced Texts, John Wiley \& Sons, Inc., New York, NY, USA, 1987.

[45] http://functions.wolfram.com/EllipticIntegrals/EllipticK/03/01/ $0003 /$.

[46] R. C. Jaeger, M. Motalab, S. Hussain, and J. C. Suhling, "Fourwire bridge measurements of silicon van der Pauw stress sensors," Journal of Electronic Packaging, Transactions of the ASME, vol. 136, no. 4, Article ID 041014, 10 pages, 2014.

[47] H. Shibata and J.-I. Oide, "Analysis of the Hall effect device using an anisotropic material," Journal of Applied Physics, vol. 88, no. 8, pp. 4813-4817, 2000.

[48] W. von Koppenfels and F. Stallmann, Praxis der Konformen Abbildung, chapter B.7, p. 318, Springer-Verlag, Berlin, Germany, 1959.

[49] http://functions.wolfram.com/09.34.16.0020.001.

[50] Y. Kanda, "Piezoresistance effect of silicon," Sensors and Actuators A: Physical, vol. 28, no. 2, pp. 83-91, 1991.

[51] R. S. Popović, "Hall-effect devices," Sensors and Actuators, vol. 17, no. 1-2, pp. 39-53, 1989.

[52] B. Hälg, "Piezo-Hall coefficients of n-type silicon," Journal of Applied Physics, vol. 64, no. 1, pp. 276-282, 1988.

[53] U. Ausserlechner, M. Motz, and M. Holliber, "Compensation of the piezo-Hall effect in integrated Hall sensors on (100)-Si," IEEE Sensors Journal, vol. 7, no. 11, pp. 1475-1482, 2007.

[54] Y. Kanda and M. Migitaka, "Effect of mechanical stress on the offset voltages of Hall devices in Si IC," Physica Status Solidi (a)-Applications and Materials Science, vol. 35, no. 2, pp. K115K118, 1976. 

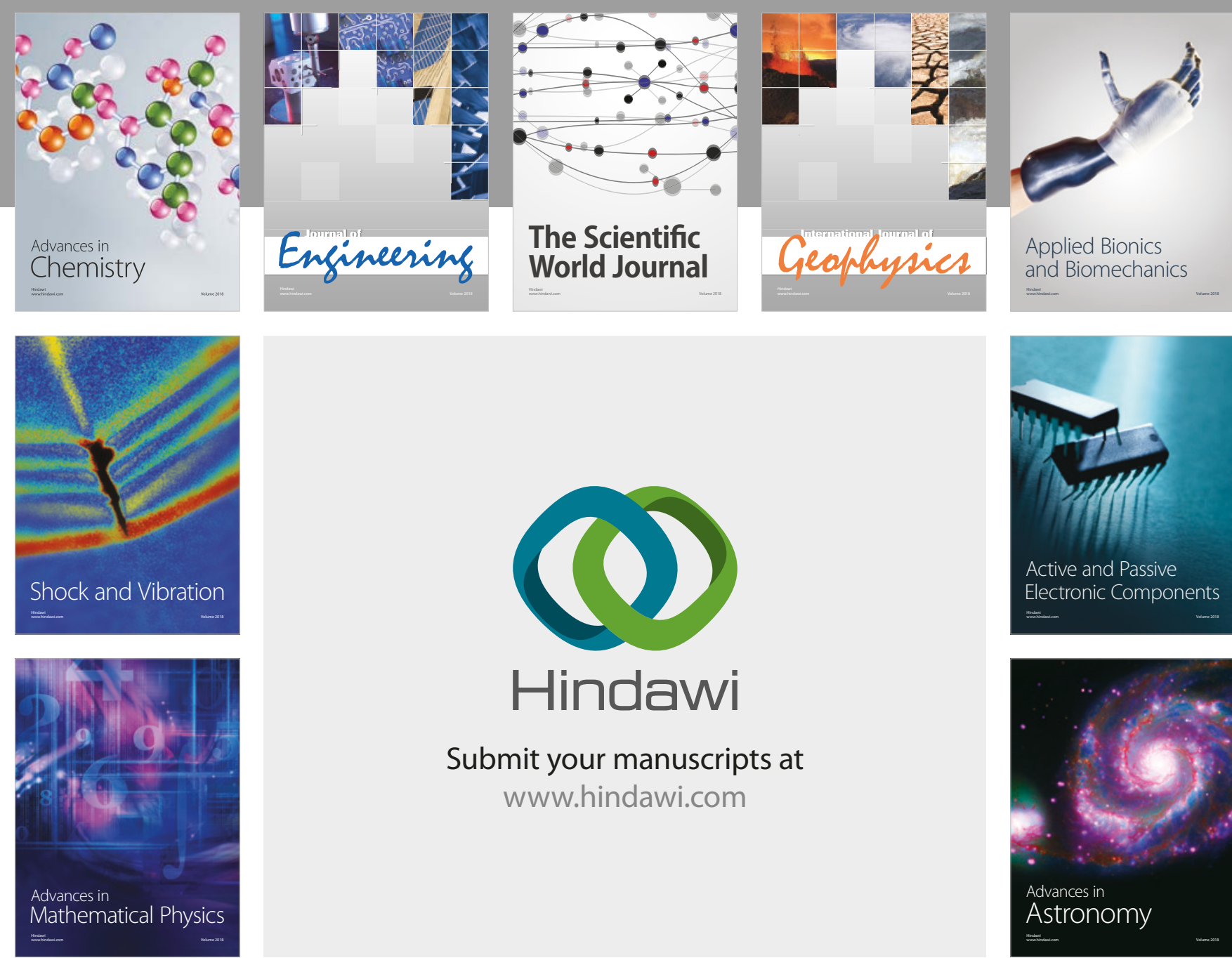

Submit your manuscripts at

www.hindawi.com

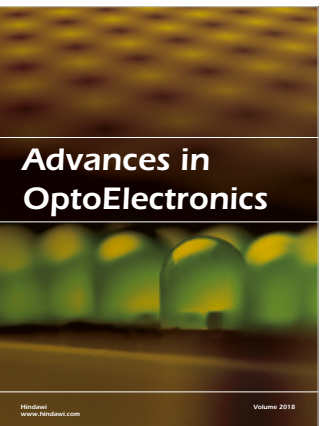

\section{Rotcting Machinery}
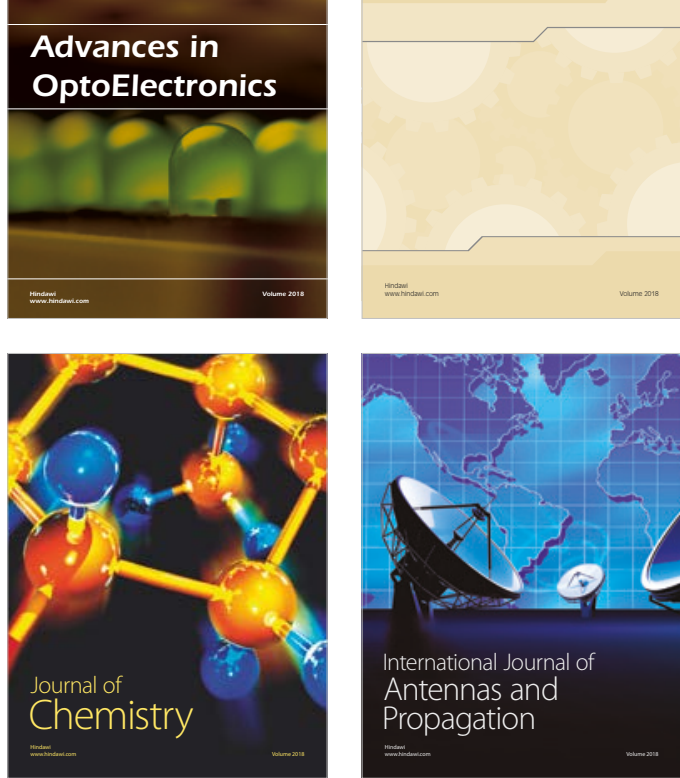

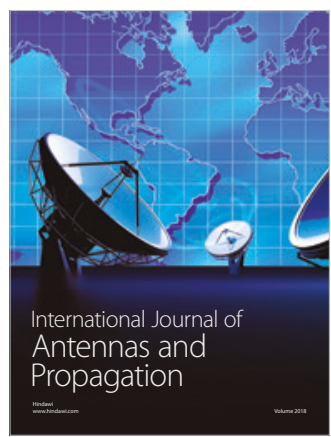

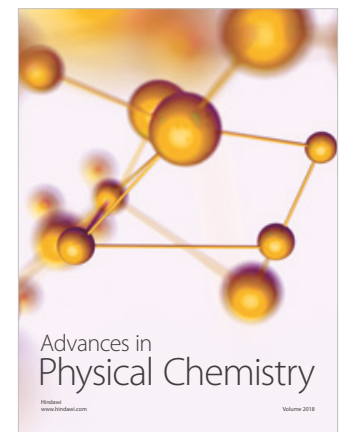

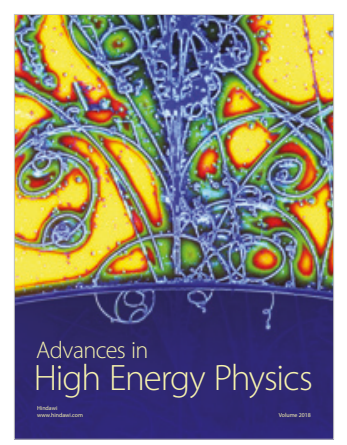

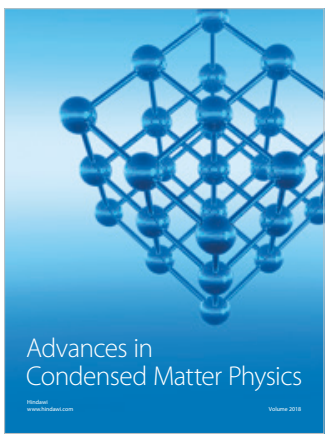

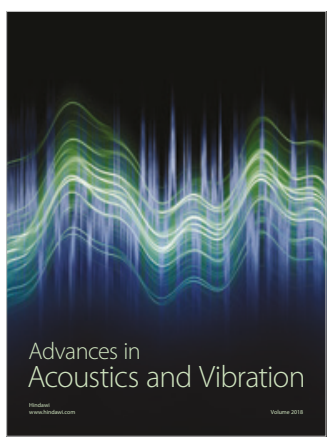

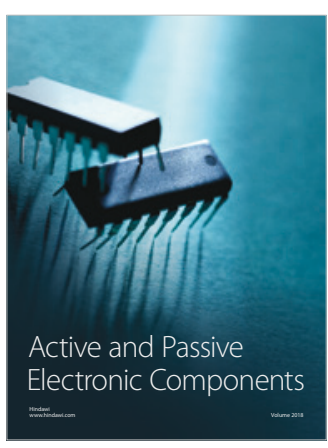
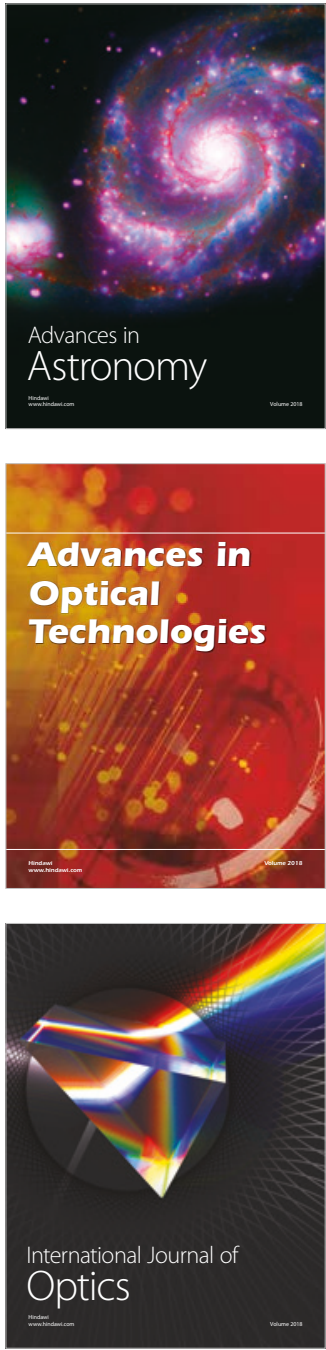